\title{
A Continuous Time Structural Model for Insolvency, Recovery, and Rollover Risks
}

\author{
Gechun Liang ${ }^{\dagger}$, Eva Lütkebohmert* ${ }^{*}$, and Wei $\mathrm{Wei}^{\ddagger}$ \\ ${ }^{\dagger}$ Oxford-Man Institute of Quantitative Finance, University of Oxford, U.K., \\ gechun.liang@oxford-man.ox.ac.uk \\ *Institute for Research in Economic Evolution, University of Freiburg, Germany, \\ eva.luetkebohmert@vwl.uni-freiburg.de \\ ${ }^{\ddagger}$ Mathematical Institute, University of Oxford, U.K., \\ wei.wei@maths.ox.ac.uk
}

December 7, 2012

This work was supported by the Oxford-Man Institute of Quantitative Finance, University of Oxford, and by the Excellence Initiative through the project "Pricing of Risk in Incomplete Markets" within the Institutional Strategy of the University of Freiburg. The financial support is gratefully acknowledged by the first and the second authors. Several helpful comments and suggestions from Lishang Jiang, Yajun Xiao, and Qianzi Zeng are very much appreciated. We also thank the participants at the Conference on Liquidity and Credit Risk in Freiburg 2012, the INFORMS International Meeting in Beijing 2012, and the 4th Berlin Workshop on Mathematical Finance for Young Researchers in Berlin 2012, as well as the seminar participants at University of Oxford, Imperial College, University of Texas at Austin, and Tongji University for several insightful remarks. 


\begin{abstract}
We propose a unified structural credit risk model incorporating insolvency, recovery and rollover risks. The firm finances itself mainly by issuing short- and long-term debt. Short-term debt can have either a discrete or a more realistic staggered tenor structure. We show that a unique threshold strategy (i.e., a bank run barrier) exists for short-term creditors to decide when to withdraw their funding, and this strategy is closely related to the solution of a non-standard optimal stopping time problem with control constraints. We decompose the total credit risk into an insolvency component and an illiquidity component based on such an endogenous bank run barrier together with an exogenous insolvency barrier.
\end{abstract}

Key words: Structural credit risk model, bank run, rollover risk, first passage time, optimal stopping time 


\section{Introduction}

The recent financial crisis has dramatically shown that financial markets are not ideal. In particular, refinancing in periods of financial distress can be extremely costly or even impossible due to liquidity drying up in the market. It has been shown, for example by Adrian and Shin $(2008,2010)$ and Brunnermeier (2009), that the heavy use of short-term debt was a key contributing factor to the credit crunch of 2007/2008. Financial institutions, however, often prefer short-term debt financing as it is cheaper than long-term debt. Moreover, as argued by He and Xiong (2012b), short-term debt can also be regarded as a disciplinary device for firms and can be used to mitigate adverse selection problems and reduce the cost of auditing firms. Hence, several reasons support the use of short-term financing. However, most of the existing credit risk models do not take into account the rollover risk inherent in short-term debt financing. It is the aim of this paper to provide a unified framework that incorporates rollover risk, as well as insolvency and recovery risks, into structural credit risk modeling.

The structural credit risk models were initiated by Merton (1974) and Black and Cox (1976). In these models, the default happens if the firm fundamental falls below some exogenous default barrier. The crucial assumption is the exogenously given default barrier that often relates to the firm's debt level. Most of the existing work on structural credit risk modeling focuses on how to model such an exogenous default barrier, as in Longstaff and Schwartz (1995) and Briys and de Varenne (1997), among others ${ }^{1}$. In the

\footnotetext{
${ }^{1}$ Optimal capital structural models are regarded as the second generation of structural credit risk models, which were initiated by Leland (1994, 1998), and Leland and Toft (1996). Therein the firm defaults when its equity value drops to zero, and the default barrier is determined endogenously by its equity holders. He and Xiong (2012a) extends this framework by including an illiquid debt market.
} 
following we will call this exogenous default barrier the insolvency barrier. In this paper we further determine an endogenous threshold value at which short-term creditors decide to withdraw their funding, i.e., to run on the bank, and we will call this barrier the bank run barrier. There is a third barrier, called the illiquidity barrier, which represents the critical value when the financial institution is unable to pay off its creditors in case of a bank run, and which is determined endogenously from the bank run barrier. In addition, we show that the bank run barrier always dominates the illiquidity barrier, which in turn dominates the insolvency barrier. This relationship among all three barriers not only helps to decompose the total credit risk into an insolvency component and an illiquidity component, but also illustrates the phenomenon that most financial institutions have defaulted due to illiquidity rather than to insolvency in the recent credit crunch.

Our first contribution is the provision of a rigorous formulation for a class of structural credit risk models that study bank runs. The classical bank run model of Diamond and Dybvig (1983) features a static setting where all the depositors simultaneously decide whether or not to withdraw their demand deposits from a solvent but illiquid bank. Ericsson and Renault (2006) and Goldstein and Pauzner (2005) provide further extensions that are, however, still in the static setting. In this paper, we consider bank runs from a dynamic viewpoint. The dynamic bank run model introduced by Morris and Shin (2010) focuses on a two-period setting where short-term creditors face a binary decision in terms of global games at an interim time point. Liang et al. (2012) provide a structural credit risk model that also takes liquidity risk into account, as short-term creditors can decide at a finite number of decision dates whether to roll over or to withdraw their funding. They derive a bank run barrier based on the comparison of binary strategies for a representative short-term creditor. Technically, the generalization from the two-period 
setting of Morris and Shin (2010) towards the multi-period setting of Liang et al. (2012) relies on the dynamic programming principle (DPP). In Liang et al. (2012) the DPP was only applied informally by comparing the expected returns for the two investment options of creditors at the rollover dates. In this paper, by introducing an appropriate value function for a representative short-term creditor, which describes the discounted expected return over the remaining rollover periods and is calculated based on the DPP, we derive the unique threshold strategy, i.e., the bank run barrier. The representative creditor decides to withdraw her funding if the firm's fundamental falls below this barrier at any decision date. In contrast to Liang et al. (2012), the corresponding dynamic programming equations presented in this paper are more generic and transparent, which in particular allows us to introduce flexible debt maturity structures into our model.

The second contribution of this paper is the imbedding of flexible debt tenor structures into structural credit risk models. In Liang et al. (2012) a discrete tenor structure is assumed such that the rollover dates of short-term debt are given by a sequence of deterministic numbers. This implies that all short-term debt expires and can be rolled over at the same time. The problem is therefore equivalent to a one-creditor problem. In reality, however, firms typically stagger the maturities for short-term debt to finance their long-term risky assets. Rollover risk is partially reduced in this way as at each maturity date only a fraction of total debt is due. Nevertheless, due to the maturity mismatch between the assets and the liabilities sides, the firm is still exposed to significant liquidity risk. Our model covers both the discrete tenor structure and the staggered tenor structure. The latter was first introduced by Leland (1994, 1998), and Leland and Toft (1996), with Hilberink and Rogers (2002) and Chen and Kou (2009) providing further technical details. The main idea is to assume a random duration of 
debt in order to reflect the maturity mismatch. Recently, He and Xiong (2012b) applied the staggered maturity structure to the bank run literature where debt maturities are modelled as arrival times of a Poisson process, whose intensity can then be interpreted as the inverse of average debt duration. In this paper, we utilize a more general and flexible Cox process to model the staggered tenor structure. The economic intuition of using the Cox maturity structure is that the average duration of the short-term debt which a firm issues should fluctuate and depend on some economic factors such as the firm fundamental or even the underlying systemic risk from the market.

The third contribution of this paper is that it answers the question whether the representative short-term creditor's rollover decision is indeed optimal. This question also arises in the existing dynamic bank run models such as those in He and Xiong (2012b), Morris and Shin (2010), and Liang et al. (2012), but has so far not been answered. In both the discrete and the staggered tenor structures, we show that the decision problem of the representative short-term creditor is equivalent to a non-standard optimal stopping time problem with control constraints. At each rollover date the representative creditor faces the risk that the firm may fail due to a bank run. If the firm survives, the creditor can then decide whether to withdraw her funding (stop) or to roll over her contract (continue). If the firm fails due to other creditors' runs, the representative creditor is forced to stop and face the recovery risk from bankruptcy. Therefore, the decision time for the representative creditor must exclude the default time due to bank runs. For the case of the staggered tenor structure, since the maturity dates are the arrival times of a Cox process, the representative creditor is only allowed to stop (i.e., to withdraw her funding) at a sequence of Cox arrival times rather than at any stopping time. In the literature, such kind of optimal stopping at Poisson-type arrival times has been used 
to solve the standard optimal stopping time problem by Krylov (2008) as the so-called randomized stopping time technique.

Finally, our fourth contribution is the incorporation of possible feedback effects of the bank run barrier on the representative creditor's beliefs about the behavior of other creditors. In Morris and Shin (2010) and Liang et al. (2012) the beliefs are modeled exogenously. The creditor is assumed to believe that the proportion of creditors not rolling over their debt is uniformly distributed. Using global games theory this assumption implies a partial equilibrium in our model. In this paper, by taking into account the possibility that the creditor might update her beliefs about the behavior of other creditors based on the derived bank run barrier, our endogenous bank run barrier can be interpreted as a general equilibrium.

The paper is organized as follows. Section 2 describes the assumptions on the firm's capital structure and explains the rollover decision of short-term creditors in the benchmark model. We also present the rigorous formulation of the rollover decision problem in terms of dynamic programming equations. In section 3 we use the creditor's value function derived in the dynamic programming equations to determine the short-term creditor's bank run barrier as well as the firm's illiquidity barrier in case of both the discrete and the staggered debt structures. We reformulate the creditor's decision problem in terms of the associated optimal stochastic control problem in section 4. Furthermore, we derive an endogenous bank run barrier and illiquidity default time in section 5 , where we take into account the fact that the creditor might update her beliefs about the behavior of other creditors. Section 6 summarizes the related literature and concludes. 


\section{Benchmark Model}

In this section, we propose a bank run model that incorporates rollover risk into the structural credit risk framework.

\subsection{Capital Structure of a Financial Institution}

Consider a financial market defined over a complete probability space $(\Omega, \mathcal{G}, \mathbf{P})$, which supports a standard Brownian motion $\left(W_{t}\right)_{t \geq 0}$ with its natural filtration $\left\{\mathcal{F}_{t}\right\}$ after augmentation.

The market interest rate $r$ is assumed to be constant. In this market, consider a financial institution, which we call a firm hereafter, whose fundamental value of assets follows

$$
\frac{d V_{t}}{V_{t}}=r_{V} d t+\sigma d W_{t},
$$

with constant volatility $\sigma>0$. The constant $r_{V}$ denotes the expected return on the firm's risky assets. We assume that the firm fundamental is publicly observable.

The firm finances its asset holdings in the duration $[0, T]$ by issuing short-term debt, such as asset-backed commercial papers and overnight repos, long-term debt such as corporate bonds, and equities and others. At initiation time $T_{0}=0$ an amount $L_{0}$ is invested long-term at rate $r_{L}$ until fixed maturity $T>0$. Moreover, an amount $S_{0}$ is invested short-term at rate $r_{S}$ until maturity $T_{1}$. When short-term debt matures it can be successively rolled over until the next rollover date. This produces a sequence of maturity dates (or rollover dates) $0=T_{0}<T_{1}<T_{2} \cdots<T_{\infty}=\infty$ for short-term debt. For the moment, we do not impose any structural conditions on the short-term debt maturities $\left\{T_{n}\right\}_{n \geq 1}$. They could be either deterministic or random. Table 1 shows the stylized balance sheet of the firm. 
Table 1: Balance sheet of the firm at time $t$

\begin{tabular}{cc}
\hline \hline Assets & Liabilities \\
\hline Firm's assets $V_{t}$ & Short-term debt $S_{t}$ \\
& Long-term debt $L_{t}$ \\
& Equities and others $E_{t}$ \\
\hline$V_{t}$ & $S_{t}+L_{t}+E_{t}$ \\
\hline
\end{tabular}

If there is no default, the value of short-term debt follows

$$
d S_{t}=r_{S} S_{t} d t
$$

and the value of long-term debt follows

$$
d L_{t}=r_{L} L_{t} d t
$$

The ratio of long-term debt over short-term debt $L_{t} / S_{t}$ is denoted by $l_{t}$ and follows

$$
d l_{t}=\left(r_{L}-r_{S}\right) l_{t} d t
$$

Moreover, we introduce a process $X_{t}$ as the ratio of the firm's asset value over the short-term debt value $X_{t}=V_{t} / S_{t}$. Hence, $X_{t}$ follows

$$
\frac{d X_{t}}{X_{t}}=\left(r_{V}-r_{S}\right) d t+\sigma d W_{t}
$$

Short-term creditors have the opportunity to withdraw their funding at the rollover dates. When the firm is under distress or when an outside investment opportunity is more attractive they will make use of this option. Long-term creditors, however, are locked in once they lend money to the firm. They are exposed to a higher risk, and therefore, should also be rewarded with a higher interest rate. Moreover, since creditors 
are exposed to the firm's default risk, a risk premium should be paid on top of the market interest rate. We have the following assumption on different interest rates.

Assumption 2.1 The long-term interest rate $r_{L}$ is strictly greater than the short-term interest rate $r_{S}$, while the latter is strictly greater than the market interest rate $r$, i.e., we assume $r_{L}>r_{S}>r$.

\subsection{The Rollover Decision of a Representative Short-Term Creditor}

Short-term creditors choose whether to renew their contracts when these expire, that is, they need to decide whether to roll over or to withdraw their funding (i.e., to run) at the maturity times.

Consider the decision problem of a representative short-term creditor. Her beliefs on whether or not the firm will survive from bank runs at each of the rollover dates $T_{n}$ is certainly one of the key factors to determine her rollover decision. Assume the creditor believes that the proportion of short-term creditors not rolling over their funding at each of the rollover dates $T_{n}$ is uniformly distributed on the interval $[0,1]$. The firm will survive bank runs if the proportion not rolling over their funding is less than

$$
\theta\left(X_{T_{n}}\right)=\min \left\{1, \frac{\psi V_{T_{n}}}{S_{T_{n}}}\right\}=\min \left\{1, \psi X_{T_{n}}\right\}
$$

where the constant $\psi$ denotes the fire-sale rate $^{2}$. Due to the uniform distribution assumption, the creditor will expect this to occur with the probability $\theta\left(X_{T_{n}}\right)$, which is called the survival probability of bank runs. This assumption on the survival probability

\footnotetext{
${ }^{2}$ The constant $\psi$ is the fire-sale rate of the firm fundamental when the firm is in a distress state, so it represents the amount that can be borrowed by pledging one unit of the risky asset as collateral. For a detailed discussion of how to endogenously determine the fire-sale rate by the leverage of the firm, we refer to Liang et al. (2012).
} 
of bank runs can be justified by arguments from global games theory even with unobservable firm fundamental for which we refer to Morris and Shin (2010). If the firm survives a bank run at maturity $T_{n}$ with probability $\theta\left(X_{T_{n}}\right)$, the creditor then faces two choices: either roll over her funding to the next maturity $T_{n+1}$ or withdraw her funding.

The second key factor to determine the representative short-term creditor's rollover decision is the insolvency risk stemming from the deterioration of the firm fundamental. To include this factor, we follow the classic first-passage-time framework (see for example Black and Cox (1976)) by assuming an exogenously given insolvency barrier

$$
D_{t}^{I n s}=S_{t} \beta\left(l_{t}\right)
$$

where $\beta:(0, \infty) \rightarrow(0, \infty)$ is a safety covenant function of the ratio $l_{t}=L_{t} / S_{t}$. As long as the asset value $V_{t}$ at any time $t$ is greater than or equal to the total value of debt $S_{t}+L_{t}$, the firm can be considered solvent. Hence, it is natural to assume that

$$
\beta\left(l_{t}\right) \leq\left(1+l_{t}\right)
$$

such that

$$
D_{t}^{I n s}=S_{t} \beta\left(l_{t}\right) \leq S_{t}\left(1+l_{t}\right)=S_{t}+L_{t}
$$

The bankruptcy time due to insolvency is then given by the following first-passage-time

$$
\tau^{I n s}=\inf \left\{t \geq 0: V_{t} \leq D_{t}^{I n s}\right\}=\inf \left\{t \geq 0: X_{t} \leq \beta\left(l_{t}\right)\right\}
$$

The third key factor for the representative short-term creditor's rollover decision is the recovery rate when the firm defaults due to either bank runs or insolvency. If the firm defaults at some time $t \in[0, T]$, the firm is exposed to certain bankruptcy costs. Suppose these are proportional to the firm fundamental value and that $\alpha V_{t}$ is the firm value after having paid the bankruptcy costs for $\alpha \in(0,1)$. Then, the value $\alpha V_{t}$ will 
be divided among all the creditors, so the representative short-term creditor obtains the proportion of her funding and she gets at most her debt value back. Thus, we define the recovery rate as

$$
R_{t}=\min \left\{1, \frac{\alpha V_{t}}{S_{t}+L_{t}}\right\}=\min \left\{1, \frac{\alpha X_{t}}{1+l_{t}}\right\}
$$

Note that in case of a default due to a bank run, it can happen that the asset value is larger than $S_{t}+L_{t}$. Therefore, we have to cut off the recovery rate by 1 . However, if the firm defaults due to insolvency at the first-passage-time $\tau^{I n s}$, the asset value by definition equals the insolvency barrier $D_{\tau^{I n s}}^{I n s}$. In this case the recovery rate equals

$$
R_{\tau^{I n s}}=\frac{\alpha D_{\tau^{I n s}}^{I n s}}{S_{\tau^{I n s}}+L_{\tau^{I n s}}}=\frac{\alpha \beta\left(l_{\tau^{I n s}}\right)}{1+l_{\tau^{I n s}}}
$$

which is less than 1 by condition (2.1) for any $\alpha \in(0,1)$.

We assume that rollover decisions are solely determined by the aforementioned factors, which we summarize as follows.

Assumption 2.2 The following three factors determine the rollover decision of a representative short-term creditor.

(i) Rollover risk is reflected by the representative short-term creditor's beliefs about the survival probability from bank runs $\theta\left(X_{T_{n}}\right)$.

(ii) Insolvency risk is reflected by the first-passage-time $\tau^{\text {Ins }}$ when the firm's asset value falls below the insolvency barrier $D_{t}^{\text {Ins }}$.

(iii) Recovery risk is reflected by the fraction $R_{t}$ of funding that the representative shortterm creditor obtains in case of a default at time $t$.

We further impose the following condition on the safety covenant function for technical convenience. 
Assumption 2.3 The safety covenant function $\beta\left(l_{t}\right)$ in the definition of the insolvency barrier $D_{t}^{\text {Ins }}$ has the linear form $\beta\left(l_{t}\right)=\beta l_{t}$ for some positive constant $\beta \leq\left(1 / l_{t}+1\right)$.

\subsection{Dynamic Programming Equations}

In this section we derive dynamic programming equations for the short-term creditor's rollover problem. We consider a representative short-term creditor who invests an amount normalized to 1 monetary unit at time $t \in[0, T]$. Her discounted expected return over the remaining time period $(t, T]$ is described by the value function $U(t, x)$ and depends on the current ratio $X_{t}=x$ of asset value over short-term debt value. Here we discount at the market rate $r$. To investigate the creditor's value function we go backwards in time starting with her last rollover date prior to terminal time $T$. Suppose that her $N^{\text {th }}$ rollover date is the closest one prior to the maturity $T$ of long-term debt, that is, $T_{N} \leq T$ and $T_{N+1} \geq T$.

At the terminal time $T$, the representative short-term creditor faces the insolvency risk that the firm may not pay back her funding, and her value function at the terminal time is ${ }^{3}$

$$
U(T, x)=R_{T}=\min \left\{1, \frac{x}{1+l_{T}}\right\} .
$$

During the last time period $\left(T_{N}, T\right)$, all the creditors are locked in, so there is no rollover risk, and the representative short-term creditor only faces the insolvency risk with the associated recovery risk. Her value function for $t \in\left(T_{N}, T\right)$ is

$$
\begin{aligned}
U(t, x)= & \mathbf{E}_{t}^{x}\left\{\mathbf{1}_{\left\{t \leq \tau^{I n s}<T\right\}} e^{-r\left(\tau^{I n s}-t\right)} \cdot e^{r_{S}\left(\tau^{I n s}-t\right)} R_{\tau^{I n s}}\right. \\
& \left.+\mathbf{1}_{\left\{\tau^{I n s} \geq T\right\}} e^{-r(T-t)} \cdot e^{r_{S}(T-t)} U\left(T, X_{T}\right)\right\}
\end{aligned}
$$

\footnotetext{
${ }^{3}$ The probability of the insolvency time $\tau^{\text {Ins }}$ equal to the terminal time $T$ is zero, so at the terminal time $T$ the firm only faces the insolvency stemming from the final workout of the firm's risky project. For this reason the recovery rate $R$ at time $T$ is redefined as $R_{T}=\min \left\{1, X_{T} /\left(1+l_{T}\right)\right\}$.
} 
where the first term in the bracket captures the insolvency risk from the firm fundamental falling below the insolvency barrier $D_{t}^{I n s}$ during the time period $(t, T)$, and the second term captures the insolvency risk from the final workout of the firm's risky project at time $T$.

To determine the value function at $t=T_{N}$ we take a closer look at the rollover decision problem. At the rollover date $T_{N}$, if the firm survives from a bank run, the representative short-term creditor will compare the expected return from rolling over her funding with the expected market return, and will choose whatever results in a higher return for her. If the firm defaults due to a bank run, she will receive the recovery value $R_{T_{N}}$ in any case, regardless of whether she decides to roll over her funding or not. Hence, the current rollover risk at rollover date $T_{N}$ will not influence her rollover decision. Therefore, the value function given in equation (2.4) also describes her discounted expected return at time $t=T_{N}$ for the remaining time period $\left(T_{N}, T\right)$.

In general, during the time period $\left[T_{n}, T_{n+1}\right)$ for $n=0,1, \ldots, N-1$, the representative short-term creditor is exposed not only to the insolvency risk arising from the deterioration of the firm fundamental in the period $\left[T_{N}, T_{n+1}\right)$ but also to the rollover risk caused by other creditors' rollover decisions at time $T_{n+1}$. Table 2 summarizes her payoff at maturity $T_{n+1}$.

Table 2: Representative creditor's aggregate payoff from $T_{n}$ to $T_{n+1}$

\begin{tabular}{cccc}
\hline \hline Decision & Solvency in $\left[T_{n}, T_{n+1}\right]$ & Solvency in $\left[T_{n}, T_{n+1}\right]$ & Insolvency in $\left[T_{n}, T_{n+1}\right]$ \\
at $T_{n+1}$ & and not successful run at $T_{n+1}$ & but successful run at $T_{n+1}$ & \\
\hline Run & $e^{r_{S}\left(T_{n+1}-T_{n}\right)} \cdot 1$ & $e^{r_{S}\left(T_{n+1}-T_{n}\right)} \cdot R_{T_{n+1}}$ & $e^{r_{S}\left(\tau^{\text {Ins }}-T_{n}\right)} R_{\tau^{\text {Ins }}}$ \\
Rollover & $e^{r_{S}\left(T_{n+1}-T_{n}\right)} \cdot U\left(T_{n+1}, X_{T_{n+1}}\right)$ & $e^{r_{S}\left(T_{n+1}-T_{n}\right)} \cdot R_{T_{n+1}}$ & $e^{r_{S}\left(\tau^{\text {Ins }}-T_{n}\right)} R_{\tau^{\text {Ins }}}$ \\
\hline
\end{tabular}


At maturity $T_{n+1}$ if there is no default, the representative short-term creditor either withdraws her funding to get $e^{r_{S}\left(T_{n+1}-T_{n}\right)} \cdot 1$ or renews her contract to receive $e^{r_{S}\left(T_{n+1}-T_{n}\right)} \cdot U\left(T_{n+1}, X_{T_{n+1}}\right)$. If the firm defaults due to a bank run at time $T_{n+1}$, the creditor just gets the fraction $R_{T_{n+1}}$ of her funding $e^{r_{S}\left(T_{n+1}-T_{n}\right)}$ back. Since the creditor believes that the firm survives a bank run at time $T_{n+1}$ with probability $\theta\left(X_{T_{n+1}}\right)$, her discounted expected return at time $t \in\left[T_{n}, T_{n+1}\right)$ over the remaining time period $[t, T]$ can be described by the following value function

$$
\begin{aligned}
U(t, x)=\mathbf{E}_{t}^{x}\left\{\mathbf{1}_{\left\{t \leq \tau^{I n s}<T_{n+1}\right\}} e^{\left(r_{S}-r\right)\left(\tau^{I n s}-t\right)} R_{\tau^{I n s}}+\mathbf{1}_{\left\{\tau^{I n s} \geq T_{n+1}\right\}} e^{\left(r_{S}-r\right)\left(T_{n+1}-t\right)} .\right. \\
\left.\cdot\left[\theta\left(X_{T_{n+1}}\right) \max \left\{1, U\left(T_{n+1}, X_{T_{n+1}}\right)\right\}+\left(1-\theta\left(X_{T_{n+1}}\right)\right) R_{T_{n+1}}\right]\right\} .
\end{aligned}
$$

The first term on the right hand side captures the insolvency risk within the time period $\left[t, T_{n+1}\right)$, whereas the second term captures the rollover risk at time $T_{n+1}$ as well as the insolvency and rollover risks in $\left[T_{n+1}, T\right]$.

The dynamic programming equations (2.4) and (2.5) for the value function $U(t, x)$ are the key drivers to determine the bank run barrier in our model, which will be discussed later. By the Feynman-Kac formula, we have the following partial differential equation (PDE) representation for the value function $U(t, x)$.

Proposition 2.1 Suppose Assumptions 2.1, 2.2, and 2.3 are satisfied. For $n=0,1, \ldots, N-$ 1 , let $W_{n}(t, x)$ be the unique solution to the following PDE Dirichlet problem on $\left[T_{n}, T_{n+1}\right] \times$ $\left[\beta l_{t}, \infty\right)$

$$
\left\{\begin{aligned}
& \frac{\partial W_{n}}{\partial t}+\mathcal{L} W_{n}+\left(r_{S}-r\right) W_{n}=0 \\
& W_{n}\left(t, \beta l_{t}\right)=\alpha \beta l_{t} /\left(1+l_{t}\right) \\
& W_{n}\left(T_{n+1}, x\right)= \theta(x) \max \left\{1, W_{n+1}\left(T_{n+1}, x\right)\right\} \\
&+(1-\theta(x)) \alpha x /\left(1+l_{T_{n+1}}\right),
\end{aligned}\right.
$$


and for $n=N$, let $W_{N}(t, x)$ be the unique solution to the following Dirichlet problem on $\left[T_{N}, T\right] \times\left[\beta l_{t}, \infty\right)$

$$
\left\{\begin{array}{l}
\frac{\partial W_{N}}{\partial t}+\mathcal{L} W_{N}+\left(r_{S}-r\right) W_{N}=0 \\
W_{N}\left(t, \beta l_{t}\right)=\alpha \beta l_{t} /\left(1+l_{t}\right) \\
W_{N}(T, x)=\min \left\{1, x /\left(1+l_{T}\right)\right\},
\end{array}\right.
$$

where $\mathcal{L}$ is the infinitesimal generator for the ratio process $X$ given by

$$
\mathcal{L}=\frac{1}{2} \sigma^{2} x^{2} \frac{\partial^{2}}{\partial x^{2}}+\left(r_{V}-r_{S}\right) x \frac{\partial}{\partial x} .
$$

Then the value function $U(t, x)$ is given by concatenating $W_{n}(t, x)$ together

$$
U(t, x)=W_{n}(t, x) \text { for } t \in\left[T_{n}, T_{n+1}\right) .
$$

Based on the Green's function technique, we further have the following analytical representation for the value function $U(t, \bar{x})$ where $\bar{x}=x /\left(\beta l_{t}\right)$.

Proposition 2.2 (Green's Representation) For $n=0,1, \ldots, N$, denote $P_{n}$ and $\Phi_{n}$ respectively as the boundary condition and the terminal condition of the corresponding PDE for the value function $U(t, \bar{x})$ on $\left[T_{n}, T_{n+1}\right)$, where $T_{N+1}=T$ for convenience. Then

$$
U(t, \bar{x})=\int_{1}^{\infty} P_{n}(\xi) \mathbb{G}\left(t, \bar{x} ; T_{n+1}, \xi\right) d \xi+\left.\frac{1}{2} \sigma^{2} \int_{t}^{T_{n+1}} \Phi_{n}(\eta) \frac{\partial}{\partial \xi}\left\{\xi^{2} \mathbb{G}(t, \bar{x} ; \eta, \xi)\right\}\right|_{\xi=1} d \eta
$$

on $\left[T_{n}, T_{n+1}\right)$, where $\mathbb{G}(t, \bar{x} ; \eta, \xi)$ is the Green's function for the operator $\mathcal{L}^{v}$ defined as

$$
\mathcal{L}^{v}=\frac{\partial}{\partial t}+\frac{1}{2} \sigma^{2} \bar{x}^{2} \frac{\partial^{2}}{\partial \bar{x}^{2}}+\left(r_{V}-r_{L}\right) \bar{x} \frac{\partial}{\partial \bar{x}}+\left(r_{S}-r\right)
$$

on the domain $\left[T_{n}, T_{n+1}\right] \times[1, \infty)$ given by

$$
\begin{aligned}
\mathbb{G}\left(t, \bar{x} ; T_{n+1}, \xi\right)= & \frac{e^{\left(r_{S}-r\right)\left(T_{n+1}-t\right)}}{\xi \sigma \sqrt{2 \pi\left(T_{n+1}-t\right)}} \exp \left\{-\frac{\left[\log \frac{\bar{x}}{\xi}+\left(r_{V}-r_{L}-\frac{1}{2} \sigma^{2}\right)\left(T_{n+1}-t\right)\right]^{2}}{2 \sigma^{2}\left(T_{n+1}-t\right)}\right\} \\
& \times\left[1-\exp \left\{\frac{2 \log \frac{1}{\xi} \log \bar{x}}{\sigma^{2}\left(T_{n+1}-t\right)}\right\}\right] .
\end{aligned}
$$


Proof. See Appendix A.1.

\section{Bank Run Barrier and Illiquidity Barrier}

In this section, we use the value function $U(t, x)$ in the dynamic programming equations (2.4) and (2.5) to determine the bank run barrier as well as the illiquidity barrier for the representative short-term creditor. Our main objective is to show the monotone relationship among the bank run barrier, the illiquidity barrier, and the exogenously given insolvency barrier.

\subsection{Discrete Tenor Structure}

In this subsection, we extend the main results in Liang et al. (2012) to our general setup. Liang et al. (2012) show that there exists a threshold, called the bank run barrier, such that the representative short-term creditor will withdraw her funding whenever the firm fundamental falls below this barrier at a rollover date. The bank run barrier is only a finite sequence of numbers, since the creditor only has a finite number of rollover dates to decide whether to run or not. In our general setting we define the bank run barrier $D_{T_{n}}^{\text {Run }}$ for any $n=0,1, \ldots, N$ as the critical asset value such that the representative short-term creditor is indifferent in terms of running or rolling over her debt, i.e., it is defined via the unique value $x_{T_{n}}^{*}$ such that $1=U\left(T_{n}, x_{T_{n}}^{*}\right)$ in the maximum term in dynamic programming equation (2.5). The bank run barrier $D_{T_{n}}^{R u n}$ is then determined by

$$
D_{T_{n}}^{\text {Run }}=x_{T_{n}}^{*} S_{T_{n}}=x_{T_{n}}^{*} S_{0} e^{r_{S} T_{n}}, \quad \text { for } n=0,1, \ldots, N .
$$

In the following, we show that such a bank run barrier always dominates the insolvency barrier. Note that the value function $U(t, x)$ is obviously increasing with respect 
to $x$, and when the firm goes bankrupt due to insolvency at a rollover date $T_{n}=\tau^{I n s}$, the value function is

$$
U\left(T_{n}, \beta l_{T_{n}}\right)=R_{T_{n}}=\alpha \beta l_{T_{n}} /\left(1+l_{T_{n}}\right)
$$

Due to Assumption 2.3 we have $\beta \leq\left(1 / l_{T_{n}}+1\right)$ so that $R_{T_{n}}=\alpha \beta l_{T_{n}} /\left(1+l_{T_{n}}\right) \leq 1$. Hence we have $\beta l_{T_{n}} \leq x_{T_{n}}^{*}$ since $U\left(T_{n}, x_{T_{n}}^{*}\right)=1$. This means the insolvency barrier $D_{t}^{I n s}$ at any rollover date $t=T_{n}$ is dominated by the bank run barrier, i.e., $D_{T_{n}}^{I n s} \leq D_{T_{n}}^{\text {Run }}$ for $n=0,1, \ldots, N$. Note that this dominance always holds in Liang et al. (2012), since the recovery rate $R_{t}$ is assumed to be zero therein.

A bank run does not necessarily trigger a default, for example in the case where the firm can raise enough funding to pay off all its creditors. We assume that in case of a bank run the firm has the option to issue collateralized debt by pledging its assets as collateral. The collateral value of the assets is expressed in terms of the fire-sale price $\psi V_{T_{n}}$ for $\psi \in(0,1)$, such that the funds that can be raised in this way are at most $\psi V_{T_{n}}$ at rollover date $T_{n}$. If $\psi V_{T_{n}} \geq S_{T_{n}}+L_{T_{n}}$ the firm can raise enough funds to pay off all its creditors, and a potential bank run at time $T_{n}$ would not lead to a default. Motivated by this observation we introduce a third barrier, which we call an illiquidity barrier and which is denoted by $D_{t}^{I l l}$ in the following. As an illiquidity default only occurs if there is a bank run and the firm is not able to raise enough funds to pay off its creditors, the illiquidity barrier is naturally defined as

$$
D_{T_{n}}^{I l l}=\min \left\{D_{T_{n}}^{\text {Run }},\left(S_{T_{n}}+L_{T_{n}}\right) / \psi\right\}, \quad \text { for } n=0,1, \ldots, N .
$$

Again by Assumption 2.3 we have $\beta \leq\left(1 / l_{t}+1\right)$ so that $\beta l_{t} S_{t} \leq\left(S_{t}+L_{t}\right)$, which means the insolvency barrier $D_{t}^{I n s}$ at $t=T_{n}$ is also dominated by the illiquidity barrier, i.e., $D_{T_{n}}^{I n s} \leq D_{T_{n}}^{I l l}$ for $n=0,1, \ldots, N$.

In summary, we have the following relationship among the three barriers. 
Theorem 3.1 Suppose that Assumptions 2.1, 2.2, and 2.3 are satisfied. Then at any maturity $T_{n}$, the bank run barrier is no less than the illiquidity barrier, while the latter is no less than the insolvency barrier, i.e.,

$$
D_{T_{n}}^{I n s} \leq D_{T_{n}}^{I l l} \leq D_{T_{n}}^{\text {Run }} \quad \text { for } n=0,1, \ldots, N
$$

Figure 1 illustrates different scenarios in our bank run model with the discrete tenor structure for three simulated asset value paths. Here we assume $N=4$ rollover dates at times $t=2,4,6$, and 8 . The dotted line shows the bank run barrier, the dashed line the illiquidity barrier, and the dashed-dotted line the insolvency barrier. Note that in this discrete setting, the bank run barrier and the illiquidity barrier are not continuous functions. They consist only of the marked points. The middle grey asset value path falls below the insolvency barrier shortly before $t=4$. At the only rollover date prior to this time, the asset value is larger than the bank run barrier. Hence, in this simulation the firm would have defaulted shortly before $t=4$ due to insolvency. The black path falls below the illiquidity barrier at the third rollover date at time $t=6$. It always stays above the insolvency barrier. Thus, in this simulation the firm defaults due to illiquidity at time $t=6$. Finally, the light grey graph shows a scenario where a bank run occurs at the last rollover date at time $t=8$. At that time, however, the asset value is still larger than the illiquidity barrier, meaning that the firm is able to raise enough funds to pay off its creditors. Hence, the firm survives the bank run.

$$
\text { [Insert Figure } 1 \text { here.] }
$$

\subsection{Staggered Tenor Structure}

In Liang et al. (2012) it is assumed that short-term debt rollover dates are given by a deterministic sequence of numbers and that they are the same for all short-term creditors. 
This assumption is rather restrictive. The firm is highly exposed to rollover risk in such a setting where all short-term funding expires at the same date. In practice, however, firms tend to spread out their debt expirations across time to reduce their exposure to liquidity risk. In this paper, we introduce a more flexible debt maturity structure. Among others, Leland $(1994,1998)$ and Leland and Toft (1996) introduced the so-called staggered maturity structure to capture this fact. The idea is to use the arrival times of a Poisson process to model the maturities of short-term debt. In other words, the duration of short-term debt $T_{1}-T_{0}, T_{2}-T_{1}, \ldots$ has an exponential distribution. While the random duration assumption appears different from the standard debt contract with a predetermined maturity, it captures the staggered debt maturity structure of a typical firm. For the application of such a Poisson maturity structure in the literature of bank runs, we refer to the recent work by He and Xiong (2012b).

The crucial parameter under the aforementioned Poisson maturity structure framework is the intensity $\lambda$. Its inverse $1 / \lambda$ can be interpreted as the average duration of short-term debt. In this section, we consider a Cox maturity structure, meaning that the maturity of short-term debt follows a more general and flexible Cox process. Recall that a Cox process is a generalization of Poisson processes in which the intensity is allowed to be random but in such a way that if we condition on a particular realization $\lambda_{t}(\omega)$ of the intensity, the process becomes an inhomogeneous Poisson process with intensity $\lambda_{t}(\omega)$. The economic intuition of using the Cox maturity structure is that the average duration of the short-term debt that the firm issues should depend on some time-dependent economic factors such as the firm fundamental $V$, the ratio $X$ of the firm fundamental over the short-term debt, or even some underlying systematic risk factors from the market. In the following we therefore assume that the average maturity is a function of the ratio process $X$. 
We construct the short-term debt maturities $\left\{T_{n}\right\}_{n \geq 1}$ by so-called canonical construction. Let $\left\{E_{n}\right\}_{n \geq 1}$ be a sequence of independent identically distributed (i.i.d.) exponential random variables on some complete probability space $(\tilde{\Omega}, \tilde{\mathcal{F}}, \tilde{\mathbf{P}})$, and define the enlarged probability space by

$$
\bar{\Omega}=\Omega \times \tilde{\Omega}, \quad \overline{\mathcal{G}}=\mathcal{F} \otimes \tilde{F}, \quad \text { and } \quad \mathbf{Q}=\mathbf{P} \otimes \tilde{\mathbf{P}} .
$$

We assume the intensity has the form $\lambda_{t}=g\left(X_{t}\right)$, where $g:(0, \infty) \rightarrow(0, \infty)$ is a smooth function with compact support. Then the maturities of short-term debt are constructed recursively as

$$
T_{0}=0 \quad \text { and } \quad T_{n}=\inf \left\{t \geq T_{n-1}: \int_{T_{n-1}}^{t} g\left(X_{s}\right) d s \geq E_{n}\right\}, \quad \text { for } n \geq 1 .
$$

The original Brownian filtration is enlarged by $\mathcal{G}_{t}=\mathcal{F}_{t} \vee \mathcal{H}_{t}$ for $t \geq 0$ with $\mathcal{H}_{t}=\sigma\left(\left\{T_{1} \leq\right.\right.$ $u\}: u \leq t)$. We summarize the above construction in the following assumption.

Assumption 3.1 (Cox maturity structure) The maturities of the short-term $\operatorname{debt}\left\{T_{n}\right\}_{n \geq 1}$ are the arrival times of a Cox process with intensity $g\left(X_{t}\right)$.

Under the Cox maturity structure, we still employ the representative short-term creditor's dynamic programming equations (2.4) and (2.5) to determine her value function $U(t, x)$. Letting the ratio process start from $X_{t}=x$ and the short-term debt maturities start from $T_{0}=t$, we synthesize the dynamic programming equations (2.4) and (2.5) for any $t \in[0, T)$ into the following succinct form

$$
\begin{aligned}
U(t, x)=\mathbf{E}_{t}^{x}\left\{\mathbf{1}_{\left\{t \leq \tau^{I n s}<T\right\}}[\right. & \mathbf{1}_{\left\{t<T_{1}<\tau^{I n s}\right\}} e^{\left(r_{S}-r\right)\left(T_{1}-t\right)}\left[\theta\left(X_{T_{1}}\right) \max \left\{1, U\left(T_{1}, X_{T_{1}}\right)\right\}+\left(1-\theta\left(X_{T_{1}}\right)\right) R_{T_{1}}\right] \\
& \left.+\mathbf{1}_{\left\{T_{1} \geq \tau^{I n s}\right\}} e^{\left(r_{S}-r\right)\left(\tau^{I n s}-t\right)} R_{\tau^{I n s}}\right] \\
+ & \mathbf{1}_{\left\{\tau^{I n s} \geq T\right\}}\left[\mathbf{1}_{\left\{t<T_{1}<T\right\}} e^{\left(r_{S}-r\right)\left(T_{1}-t\right)}\left[\theta\left(X_{T_{1}}\right) \max \left\{1, U\left(T_{1}, X_{T_{1}}\right)\right\}+\left(1-\theta\left(X_{T_{1}}\right)\right) R_{T_{1}}\right]\right. \\
& \left.\left.+\mathbf{1}_{\left\{T_{1} \geq T\right\}} e^{\left(r_{S}-r\right)(T-t)} \min \left\{1, X_{T} /\left(1+l_{T}\right)\right\}\right]\right\}
\end{aligned}
$$


Using properties for the first arrival time (i.e., the first short-term debt maturity) $T_{1}$ and the intensity $g\left(X_{t}\right)$, and applying the Feynman-Kac formula, we derive the following PDE representation for the value function $U(t, x)$ under the Cox maturity structure.

Proposition 3.2 Suppose that Assumptions 2.1, 2.2, 2.3 and 3.1 are satisfied. Then the value function $U(t, x)$ satisfies the following semi-linear PDE Dirichlet problem on $[0, T] \times\left[\beta l_{t}, \infty\right):$

$$
\left\{\begin{array}{l}
\frac{\partial U}{\partial t}+\mathcal{L} U+\left(r_{S}-r-g(x)\right) U \\
\quad+g(x)\left[\theta(x) \max \{1, U\}+(1-\theta(x)) \alpha x /\left(1+l_{t}\right)\right]=0 \\
U\left(t, \beta l_{t}\right)=\alpha \beta l_{t} /\left(1+l_{t}\right) \\
U(T, x)=\min \left\{1, x /\left(1+l_{T}\right)\right\}
\end{array}\right.
$$

Proof. See Appendix A.2.

In Appendix B, we provide a numerical algorithm to approximate the solution of the above PDE (3.3). In the rest of this section, we show that PDE (3.3) implies a unique threshold for the representative short-term creditor, i.e., there exists a unique bank run barrier $D_{t}^{\text {Run }}$ such that she will run on the firm whenever both the firm's asset value falls below such a barrier and her contract expires at some maturity $T_{n}$. Thus, the bank run time in our model is characterized endogenously by the following first-passage-time

$$
\tau^{\text {Run }}=\inf \left\{T_{n}: X_{T_{n}} \leq x^{*}\left(T_{n}\right)\right\} \wedge T,
$$

where $x^{*}(t)$ is the threshold we shall derive in the remainder of this section. Recall that $X_{t}=V_{t} / S_{t}$ is the ratio of the firm fundamental over the short-term debt, so the bank run barrier $D_{t}^{\text {Run }}$ should be given as

$$
D_{t}^{\text {Run }}=x^{*}(t) S_{t}=x^{*}(t) S_{0} e^{r_{S} t} .
$$


We derive a free-boundary problem to determine first the threshold $x^{*}(t)$ and secondly the bank run barrier $D_{t}^{\text {Run }}$ based on the semi-linear PDE (3.3).

(i) If $x>x^{*}(t)$, the representative short-term creditor will keep lending her money to the firm because either the debt is not due yet or if the debt is due she decides to roll over her funding. Her value function $U(t, x)>1$, and (3.3) reduces to

$$
\frac{\partial U}{\partial t}+\mathcal{L} U+\left(r_{S}-r-g(x)\right) U+g(x) \theta(x) U+g(x)(1-\theta(x)) \alpha x /\left(1+l_{t}\right)=0 .
$$

The third term in the above equation represents the creditor's premium of the return, the fourth term represents the expected effect of the rollover risk if the creditor rolls over her funding, and last term represents the expected effect of recovery risk associated with a potential bank run.

(ii) If $x<x^{*}(t)$, the representative short-term creditor will run on the firm if the debt is due. Her value function $U(t, x)<1$, and (3.3) reduces to

$$
\frac{\partial U}{\partial t}+\mathcal{L} U+\left(r_{S}-r-g(x)\right) U+g(x) \theta(x)+g(x)(1-\theta(x)) \alpha x /\left(1+l_{t}\right)=0 .
$$

While the third term and the last term in (3.5) have the same meanings as those in (3.4), the fourth term captures the expected effect of rollover risk from the representative short-term creditor's own run.

(iii) Finally, by the continuity of $U(t, x)$, the creditor's value function $U(t, x)$ at the threshold $x^{*}(t)$ should be equal to 1 , and the following smooth-pasting condition should be satisfied

$$
U_{x+0}\left(t, x^{*}(t)\right)=U_{x-0}\left(t, x^{*}(t)\right)
$$

In summary, we obtain the following two-phase free-boundary problem to determine the threshold (i.e., the bank run barrier) of the representative short-term creditor. 
Proposition 3.3 Suppose that Assumptions 2.1, 2.2, 2.3, and 3.1 are satisfied. Then the bank run barrier $D_{t}^{\text {Run }}$ is given by

$$
D_{t}^{\text {Run }}=x^{*}(t) S_{0} e^{r_{S} t}
$$

where $x^{*}(t)$ is the free-boundary of the following two-phase free-boundary problem

$$
\begin{cases}\frac{\partial U}{\partial t}+\mathcal{L} U+\left(r_{S}-r-g(x)\right) U+g(x) \theta(x) U+g(x)(1-\theta(x)) \alpha x /\left(1+l_{t}\right)=0, & \text { for } x>x^{*}(t), \\ U(t, x)>1, & \text { for } x>x^{*}(t), \\ U(t, x)=1, & \text { for } x=x^{*}(t), \\ U_{x}(t, x) \text { is continuous, } & \text { for } x=x^{*}(t), \\ \frac{\partial U}{\partial t}+\mathcal{L} U+\left(r_{S}-r-g(x)\right) U+g(x) \theta(x)+g(x)(1-\theta(x)) \alpha x /\left(1+l_{t}\right)=0, & \text { for } \beta l_{t}<x<x^{*}(t), \\ U(t, x)<1, & \text { for } \beta l_{t}<x<x^{*}(t), \\ U(t, x)=\alpha \beta l_{t} /\left(1+l_{t}\right), & \text { for } x=\beta l_{t},\end{cases}
$$

Proof. We only need to prove the smooth-pasting condition, which is straightforward since PDE (3.3) admits a unique classical solution.

Similar to the case of the discrete tenor structure as in section 3.1, a bank run does not necessarily trigger the firm's default. Bank runs are not successful if the firm can raise enough funding to pay off all its creditors, i.e., if the fire-sale price of the firm's asset satisfies $\psi V_{T_{n}} \geq S_{T_{n}}+L_{T_{n}}$. Therefore, we define the firm's illiquidity barrier as

$$
D_{t}^{I l l}=\min \left\{D_{t}^{\text {Run }},\left(S_{t}+L_{t}\right) / \psi\right\} \quad \text { for } t \in[0, T),
$$


and we have a similar relationship among the barriers as in the case of the discrete tensor structure.

Theorem 3.4 Suppose that Assumptions 2.1, 2.2, 2.3, and 3.1 are satisfied. Then at any time $t \in[0, T)$, the bank run barrier is greater than or equal to the illiquidity barrier, while the latter is greater than or equal to the insolvency barrier

$$
D_{t}^{I n s} \leq D_{t}^{I l l} \leq D_{t}^{\text {Run }} \quad \text { for } t \in[0, T) .
$$

The above relationship gives us the following four possible scenarios at any rollover date $T_{n}$.

(i) $V_{T_{n}} \leq D_{T_{n}}^{\text {Ins }}:$ Default due to insolvency;

(ii) $D_{T_{n}}^{I n s}<V_{T_{n}} \leq D_{T_{n}}^{I l l}$ : Bank run occurs and triggers a default due to illiquidity;

(iii) $D_{T_{n}}^{\text {Ill }}<V_{T_{n}} \leq D_{T_{n}}^{\text {Run }}$ : Bank run occurs, but not a successful run;

(iv) $D_{T_{n}}^{\text {Run }}<V_{T_{n}}$ : The creditor rolls over to the next maturity $T_{n+1}$.

Proof. The proof is essentially the same as the proof for Theorem 3.1, so we omit it.

Figure 2 illustrates different scenarios in our bank run model with a staggered tenor structure for three simulated asset value paths. Here the intensity of the Cox process is chosen to be $g(x)=0.4$. The dotted line shows the bank run barrier, the dashed line the illiquidity barrier and the dashed-dotted line the insolvency barrier, all of which are continuous functions in this model setting. The marked times $T_{1}, T_{2}$, and $T_{3}$ are the arrival times of the Cox process, which are smaller than the final date $T=10$. At these times the representative short-term creditor can decide whether or not to roll over her funding. At the first rollover date $T_{1}$ all three asset value paths are above the bank run 
barrier. Hence, the creditor decides to roll over her contract. The middle grey asset value path falls below the insolvency barrier shortly before the second rollover date $T_{2}$. Hence, the firm will default at that time point, and the creditor is left with the corresponding recovery value. At the second rollover date $T_{2}$, the light grey asset value path falls below the bank run barrier but is still above the illiquidity barrier. This means that a bank run occurs at that date, but the firm is able to pay off its creditors and survives. The black asset value path is above the bank run barrier at $T_{2}$. At the last rollover date $T_{3}$ the middle grey and the black asset value paths are below the illiquidity barrier, which means that a bank run occurs and the firm is not able to pay off all its creditors, such that the bank run actually triggers an illiquidity default and the creditor receives the corresponding recovery value. Note that all three paths fall below the bank run and the illiquidity barriers already much earlier in time. However, as these times are not rollover dates for the representative short-term creditor, she cannot withdraw her funding at those dates.

The figure also illustrates the relation between different barriers, which has been theoretically proved in Theorem 3.4; the bank run barrier is always greater than or equal to the illiquidity barrier, which in turn is always greater than or equal to the insolvency barrier.

\section{[Insert Figure 2 here.]}

Table 3 shows the frequency of illiquidity defaults, insolvency defaults, and survival scenarios out of 10000 asset value simulations in case of the staggered tenor structure model for different initial asset values $V_{0}$. The parameters used are the same as for Figure 2. The second row provides the number of simulations leading to an illiquidity default, whereas the fourth row gives the number of insolvency default simulations where the initial asset value varies from 10 to 22 . Moreover, the table states the number of 
simulations where the firm survives, which are distinguished between the situation where a bank run occurs but does not trigger an illiquidity default (3rd row) and those scenarios where the firm's asset value always stays above the bank run barrier (5th row). The results show that the number of illiquidity defaults and the total number of bank runs decrease in initial asset value as one would expect. Analogously, insolvency defaults become less likely with increasing $V_{0}$. Hence, the firm is more likely to survive with higher initial asset value $V_{0}$.

Table 3: Simulations of different scenarios in the staggered tenor structure model

Of a total number of 10000 simulations and for different initial asset values $V_{0}$, the table provides the number of simulations leading to an illiquidity default (2nd row) or to an insolvency default (4th row) as well as the number of simulations where the firm survives, which are distinguished between the situation where a bank run occurred but did not trigger an illiquidity default (3rd row) and the scenarios where the firm's asset value always stayed above the bank run barrier (5th row). Parameters are the same as in Figure 2.

\begin{tabular}{|l|c|c|c|c|c|c|c|}
\hline $\begin{array}{l}\text { Illiquidity, Run } \\
\text { and Insolvency }\end{array}$ & 10 & 12 & 14 & 16 & 18 & 20 & 22 \\
\hline Illiquidity & 5456 & 5126 & 4693 & 4491 & 4149 & 3757 & 3612 \\
\hline Run & 2641 & 2387 & 2266 & 2021 & 1934 & 1793 & 1694 \\
\hline Insolvency & 298 & 256 & 238 & 204 & 176 & 159 & 133 \\
\hline None of them & 1605 & 2231 & 2803 & 3284 & 3741 & 4291 & 4561 \\
\hline
\end{tabular}

\section{Optimal Stochastic Control Formulation}

In this section we are concerned with whether the representative short-term creditor's decision is optimal. Intuitively, since the creditor's decision follows the DPP, her decision 
should be optimal. The question then is, what is the corresponding optimal stochastic control problem? To answer this question we first investigate the case of the discrete tenor structure and then discuss the staggered debt structure.

\subsection{Optimal Stopping Time with Control Constraints}

Let us first consider the case of the discrete tenor structure, i.e., short-term debt maturities $\left\{T_{n}\right\}_{n \geq 1}$ are a sequence of deterministic numbers. Recall that at each rollover date $T_{n}$, the creditor believes that there is a probability $\left(1-\theta\left(X_{T_{n}}\right)\right)$ that the firm may default due to a successful bank run. Let $T_{*}$ denote the time that the firm defaults due to a bank run. Hence, $T_{*}$ is a random time taking value in $\left\{T_{n}\right\}_{n \geq 1}$.

Let $\tau \in\left\{T_{n}\right\}_{n \geq 1} \backslash T_{*}$ be the time at which the representative short-term creditor decides to withdraw her funding and to run on the firm. This is an $\mathcal{F}_{t^{-}}$-stopping time. We first consider the case $\left\{\tau^{I n s}<T\right\}$, i.e., the firm fails due to insolvency before its project expires. If $\tau<T_{*} \wedge \tau^{\text {Ins }}$, the creditor withdraws her funding before a successful bank run or an insolvency happens. In this case she will obtain the payoff

$$
\mathbf{1}_{\left\{\tau<T_{*} \wedge \tau^{I n s}\right\}} e^{r_{S} \tau}
$$

If $T_{*}<\tau \wedge \tau^{\text {Ins }}$, the firm fails due to a successful bank run before the creditor decides to withdraw her money and before an insolvency happens. Hence, the creditor will obtain the payoff

$$
\mathbf{1}_{\left\{T_{*}<\tau \wedge \tau^{I n s}\right\}} e^{r_{S} T_{*}} R_{T_{*}} .
$$

Finally, if $\tau^{I n s} \leq T_{*} \wedge \tau$, the firm defaults due to insolvency before a successful bank run takes place and before the creditor decides to withdraw her funding. Then, the creditor will obtain the payoff

$$
\mathbf{1}_{\left\{\tau^{I n s} \leq T_{*} \wedge \tau\right\}} e^{r_{S} \tau^{I n s}} R_{\tau^{I n s}} .
$$


On the other hand, on the event $\left\{\tau^{I n s} \geq T\right\}$, i.e., no insolvency happens before the project ends, the creditor will obtain the payoff

$$
\mathbf{1}_{\left\{\tau<T_{*} \wedge T\right\}} e^{r_{S} \tau}+\mathbf{1}_{\left\{T_{*}<\tau \wedge T\right\}} e^{r_{S} T_{*}} R_{T_{*}}+\mathbf{1}_{\left\{T \leq T_{*} \wedge \tau\right\}} e^{r_{S} T} \min \left\{1, X_{T} /\left(1+l_{T}\right)\right\} .
$$

Table 4 summarizes the aggregate payoff of the representative creditor.

Table 4: Representative creditor's aggregate payoff

\begin{tabular}{ccc}
\hline \hline Insolvency time $\tau^{\text {Ins }}$ & Decision time $\tau$ & Payoff \\
\hline$\tau^{\text {Ins }}<T$ & $\tau<T_{*} \wedge \tau^{\text {Ins }}$ & $e^{r_{S} \tau} \cdot 1$ \\
& $T_{*}<\tau \wedge \tau^{\text {Ins }}$ & $e^{r_{S} T_{*}} \cdot R_{T_{*}}$ \\
& $\tau^{\text {Ins }} \leq T_{*} \wedge \tau$ & $e^{r_{S} \tau^{I n s}} \cdot R_{\tau^{I n s}}$ \\
\hline$\tau^{\text {Ins }} \geq T$ & $\tau<T_{*} \wedge \tau^{\text {Ins }}$ & $e^{r_{S} \tau} \cdot 1$ \\
& $T_{*}<\tau \wedge T$ & $e^{r_{S} T_{*}} \cdot R_{T_{*}}$ \\
& $T \leq T_{*} \wedge \tau$ & $e^{r_{S} T} \cdot \min \left\{1, X_{T} /\left(1+l_{T}\right)\right\}$ \\
\hline
\end{tabular}

For any $0 \leq t \leq \hat{t} \leq T$, we define the aggregate discounted payoff from time $t$ to $\hat{t}$ $\operatorname{as}^{4}$

$\mathcal{A}_{t, \hat{t}}=\mathbf{1}_{\left\{t<\tau<T_{*} \wedge \hat{t}\right\}} e^{\left(r_{S}-r\right)(\tau-t)}+\mathbf{1}_{\left\{t<T_{*}<\tau \wedge \hat{t}\right\}} e^{\left(r_{S}-r\right)\left(T_{*}-t\right)} R_{T_{*}}+\mathbf{1}_{\left\{t<\hat{t} \leq T_{*} \wedge \tau\right\}} e^{\left(r_{S}-r\right)(\hat{t}-t)} R_{\hat{t}}$.

The creditor will choose an optimal $\mathcal{F}_{t}$-stopping time to maximize her expected payoff

$$
\sup _{\tau \in\left\{T_{n}\right\}_{n \geq 1} \backslash T_{*}} \mathbf{E}_{0}^{x}\left\{\mathbf{1}_{\left\{\tau^{I n s}<T\right\}} \cdot \mathcal{A}_{0, \tau^{I n s}}+\mathbf{1}_{\left\{\tau^{I n s} \geq T\right\}} \cdot \mathcal{A}_{0, T}\right\} .
$$

We have the following theorem that shows the creditor's value function $U(t, x)$ defined by the dynamic programming equations (2.4) and (2.5) is indeed optimal.

\footnotetext{
${ }^{4}$ Recall that $R_{T}=\min \left\{1, X_{T} /\left(1+l_{T}\right)\right\}$ as defined in (2.3).
} 
Theorem 4.1 The value of the optimal stopping time problem (4.1) is given by the value function $U(0, x)$ in the dynamic programming equation (2.5). The optimal stopping time is given by the earliest maturity date at which the firm fundamental falls below the bank run barrier determined in section 3.1, i.e.,

$$
\tau^{\text {Run }}=\inf \left\{T_{n}: V_{T_{n}} \leq D_{T_{n}}^{R u n}, n=0,1, \ldots, N\right\} \wedge T
$$

Proof. See Appendix A.3.

Next we consider the case of the staggered tenor structure, i.e., the maturities $\left\{T_{n}\right\}_{n \geq 1}$ are the arrival times of a Cox process with intensity $g\left(X_{t}\right)$. Similar to the discrete tenor structure, we define $T_{*}$ as the default time due to a successful bank run. Since $T_{*}$ is chosen among the Cox arrival times $\left\{T_{n}\right\}_{n \geq 1}$ with probability $\left(1-\theta\left(X_{T_{n}}\right)\right)$, it is well known that $T_{*}$ is the first arrival time of another Cox process with intensity $g\left(X_{t}\right)\left(1-\theta\left(X_{t}\right)\right)$. Let $\tau \in\left\{T_{n}\right\}_{n \geq 1} \backslash T_{*}$ again denote the rollover date at which the representative short-term creditor decides to withdraw her funding and to run on the firm. This is a $\mathcal{G}_{t}=\mathcal{F}_{t} \vee \mathcal{H}_{t}$-stopping time under the staggered tenor structure, i.e., $\tau$ must be chosen from the arrival times of the Cox process.

The representative short-term creditor will choose an optimal $\mathcal{G}_{t}$-stopping time to maximize her expected payoff

$$
\sup _{\tau \in\left\{T_{n}\right\}_{n \geq 1} \backslash T_{*}} \mathbf{E}_{0}^{x}\left\{\mathbf{1}_{\left\{\tau^{I n s}<T\right\}} \cdot \mathcal{A}_{0, \tau^{I n s}}+\mathbf{1}_{\left\{\tau^{I n s} \geq T\right\}} \cdot \mathcal{A}_{0, T}\right\}
$$

In contrast to the previous section on the discrete tenor structure, the optimal stopping time problem (4.2) can now only be stopped at the Cox random times $\left\{T_{n}\right\}_{n \geq 1} \backslash T_{*}$. Hence, knowing only the Brownian filtration $\left\{\mathcal{F}_{t}\right\}$ is certainly not enough to decide when to stop; one has to know the additional filtration $\mathcal{H}_{t}$ from the Cox process in order to determine when to stop. Similar to the case of the discrete tenor structure, we can 
show that the solution to this optimal stopping time problem is given by the dynamic programming equation (3.2).

Theorem 4.2 The value of the optimal stopping time problem (4.2) is given by the value function $U(0, x)$ in the dynamic programming equation (3.2). The optimal stopping time is given by the earliest maturity date such that the firm fundamental falling below the bank run barrier in Proposition 3.3:

$$
\tau^{\text {Run }}=\inf \left\{T_{n}: V_{T_{n}} \leq D_{T_{n}}^{\text {Run }}\right\} \wedge T
$$

Proof. See Appendix A.4.

\subsection{Comparison of the Discrete and the Staggered Tenor Structures}

This section compares different debt tenor structures, i.e., the discrete and the staggered tenor structures. A natural question to ask is, what will happen with the discrete tenor structure when the number of rollover dates increases to infinity, meaning that creditors can decide to roll over or to withdraw their funding at any time $t \in[0, T]$ ? Intuitively, one would expect that with increasing rollover frequency, one should approximate the staggered tenor structure model. However, there is another important difference between the two debt tenor structures. In the case of the discrete tenor structure we implicitly assume that all creditors have the same rollover dates, whereas in the staggered tenor structure model at each rollover date, corresponding to a Cox arrival time, only a fraction of total debt is due. Different short-term creditors hence have different rollover dates in that situation.

In the following, we will first study the impact of the intensity $\lambda_{t}=g\left(X_{t}\right)$ of the Cox process on the creditor's value function. We assume the function $g\left(X_{t}\right)$ to be constant and thus independent of the ratio process $X_{t}$. The intensity of the Cox process not 
only specifies the creditor's rollover dates but also affects the average duration of shortterm debt. For $g\left(X_{t}\right) \equiv g \in \mathbb{R}_{+}$the average duration of debt is equal to $1 / g$, and in an infinitesimal time interval $[t, t+d t]$ a fraction $g d t$ of debt is maturing. The larger $g$ that is chosen, the more debt is maturing at the same rollover date and the larger is the rollover frequency of short-term debt. Therefore, for large enough $g$ the staggered tenor structure model and the discrete tenor structure model should result in approximately the same value function $U(t, x)$ for the short-term debt. This result is empirically validated in Figure 3. The number of rollover dates in the discrete tenor structure model is fixed at $N=1000$, and the intensity of the Cox process in the staggered tenor structure model varies from $g=0.2$ to $g=200$.

[Insert Figure 3 here.]

In section 3.1, we derived the bank run barrier for the discrete tenor structure by determining the threshold ratio $x^{*}$ such that $U\left(t, x^{*}(t)\right)=1$, i.e., the creditor is indifferent on whether to roll over or to withdraw her funding. Similarly in Proposition 3.3, we derived the bank run barrier for the staggered tenor structure by solving the free-boundary problem (3.6). Next, we will investigate in Figure 4 the impact of the Cox intensity $g$ and the rollover frequency $N$ on the bank run barrier. The graphs show that the discrete tenor structure model with high rollover frequency $N$ approximates the staggered tenor structure model with large intensity $g(x)$.

[Insert Figure 4 here.]

\section{The Survival Probability of Bank Runs}

In our benchmark model, the survival probability of bank runs $\theta\left(X_{T_{n}}\right)=\min \left\{1, \psi X_{T_{n}}\right\}$ at each maturity date $T_{n}$ is given exogenously based on the assumption that each short- 
term creditor believes that the proportion of short-term creditors not rolling over their funding is uniformly distributed on the interval $[0,1]$. This is also the crucial assumption in the bank run models of Morris and Shin (2010) and Liang et al. (2012). The merit of this assumption is that the system is then an open loop.

Recall that in this open loop system, the bank run barrier is given by $D_{T_{n}}^{R u n}=x_{T_{n}}^{*} S_{T_{n}}$, where $x_{T_{n}}^{*}$ is determined by letting the value function $U\left(T_{n}, x_{T_{n}}^{*}\right)$ in Proposition 2.1 be equal to 1 . The illiquidity barrier is then given by $D_{T_{n}}^{I l l}=\min \left\{D_{T_{n}}^{R u n},\left(S_{T_{n}}+L_{T_{n}}\right) / \psi\right\}$. Both of the barriers depend on the survival probability of bank runs $\theta\left(X_{T_{n}}\right)$ but not vice versa. Algorithm 5.1 illustrates how the bank run barrier and the illiquidity barrier are determined in this bank run model.

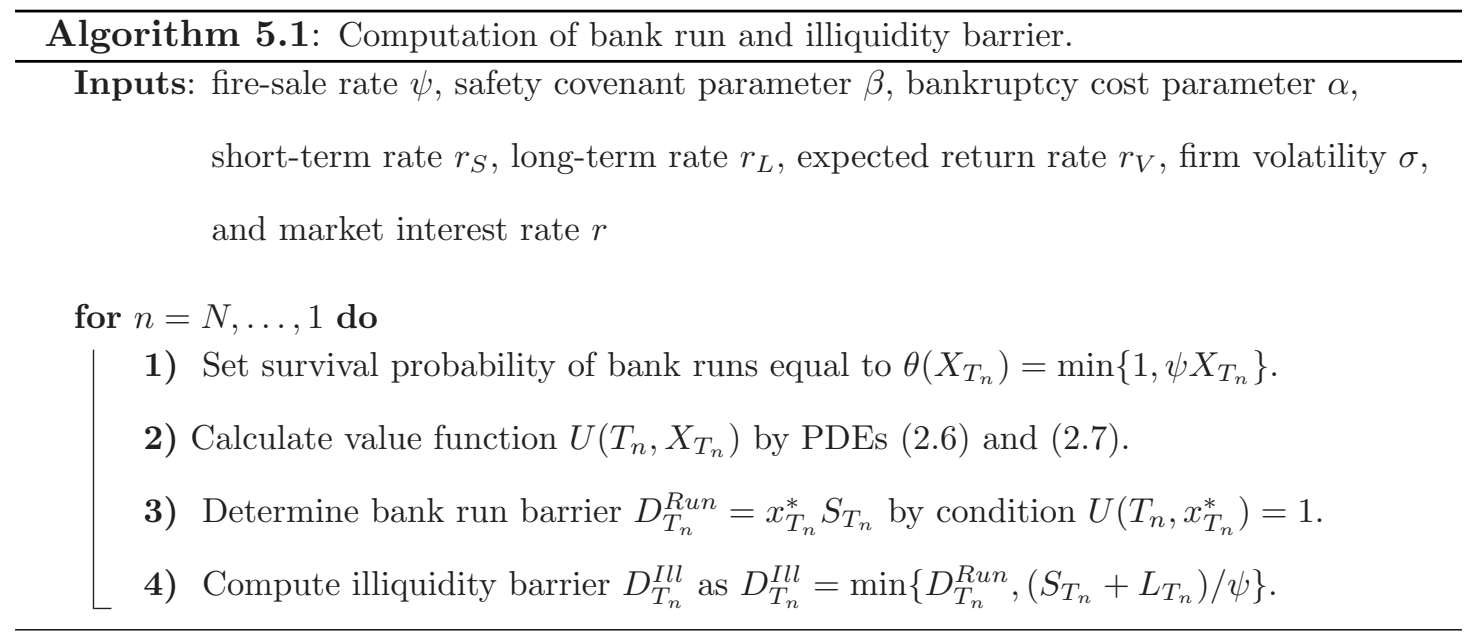

The likelihood $\theta\left(X_{T_{n}}\right)$, which the representative creditor assigns to the firm's survival probability of bank runs, is determined solely from the current ratio of asset value over short-term debt $X_{T_{n}}$. Since the representative creditor determines at each rollover date a threshold value at which she decides to withdraw her money, it seems natural to investigate whether there is a feedback effect from this illiquidity barrier on the survival probability $\theta$ of bank runs. In the following we discuss how the representative short- 
term creditor can update her beliefs about the survival probability of bank runs $\theta\left(X_{T_{n}}\right)$ based on the derived illiquidity barrier $D_{T_{n}}^{I l l}$ at each rollover date, i.e., we investigate the feedback effect between the survival probability of bank runs and both the bank run barrier and the illiquidity barrier. The system then becomes a closed loop and allows to endogenously determine the time $\tau^{I l l}=T_{*}$ of a successful bank run.

We only discuss the discrete tenor structure; the staggered tenor structure can be analyzed in the same spirit, but it involves additional technical difficulties. We drop the uniform distribution assumption on the proportion of short-term creditors not rolling over their debt, and assume that the survival probability of bank runs is a function of time $t$ and ratio $X_{t}$, i.e., $\theta(\cdot, \cdot):[0, T] \times(0, \infty) \rightarrow[0,1]$, which is to be determined. The corresponding illiquidity barrier and the bank run barrier are denoted by $\bar{D}_{T_{n}}^{I l l}$ and $\bar{D}_{T_{n}}^{R u n}$, respectively.

We again proceed backwards to determine the survival probability of bank runs. First, we define the ratio of the illiquidity barrier over the short-term debt as

$$
\bar{x}_{T_{n}}^{I l l}=\bar{D}_{T_{n}}^{I l l} / S_{T_{n}}=\min \left\{\bar{x}_{T_{n}}^{*},\left(1+l_{T_{n}}\right) / \psi\right\}, \quad \text { for } n=0,1, \ldots, N
$$

where $\bar{x}_{T_{n}}^{*}=\bar{D}_{T_{n}}^{\text {Run }} / S_{T_{n}}$. Since there is no rollover risk in the last time period $\left[T_{N}, T\right)$, the ratio of the illiquidity barrier over short-term debt at the last rollover date $T_{N}$ is independent of the survival probability of bank runs, and should be the same as the one in the open loop system

$$
\bar{x}_{T_{N}}^{I l l}=\min \left\{\bar{x}_{T_{N}}^{*},\left(1+l_{T_{N}}\right) / \psi\right\}=\min \left\{x_{T_{N}}^{*},\left(1+l_{T_{N}}\right) / \psi\right\}
$$

where $x_{T_{N}}^{*}$ is calculated as the solution of PDE (2.7). The survival probability of bank runs at time $T_{N}$ is then given by

$$
\theta\left(T_{N}, x\right)=\mathbf{E}_{T_{N}}^{x}\left\{\mathbf{1}_{\left\{X_{T_{N}} \geq \bar{x}_{T_{N}}^{I l l}\right\}}\right\}=\mathbf{1}_{\left\{x \geq \bar{x}_{T_{N}}^{I l l}\right\}} .
$$


Based on the above updated survival probability of bank runs, we calculate the ratio of the illiquidity barrier over short-term debt at the last but one rollover date $T_{N-1}$ as

$$
\bar{x}_{T_{N-1}}^{I l l}=\min \left\{\bar{x}_{T_{N-1}}^{*},\left(1+l_{T_{N-1}}\right) / \psi\right\}
$$

where $\bar{x}_{T_{N-1}}^{*}$ is calculated as the solution of PDE (2.6) but with the updated survival probability of bank runs $\theta\left(T_{N}, x\right)$ in the terminal condition. The survival probability of bank runs at the last but one rollover date $T_{N-1}$ is then given by

$$
\theta\left(T_{N-1}, x\right)=\mathbf{E}_{T_{N-1}}^{x}\left\{\mathbf{1}_{\left\{X_{T_{N-1}} \geq \bar{x}_{T_{N-1}}^{I l l}\right\}}\right\}=\mathbf{1}_{\left\{X_{T_{N-1}} \geq \bar{x}_{T_{N-1}}^{I l l}\right\}}
$$

In general, the survival probability of bank runs and the illiquidity barrier are computed backwards recursively for $n=0,1, \ldots, N-1$, according to the following procedure.

(i) Compute the ratio of the illiquidity barrier over short-term debt

$$
\bar{x}_{T_{n}}^{I l l}=\min \left\{\bar{x}_{T_{n}}^{*},\left(1+l_{T_{n}}\right) / \psi\right\}
$$

where $\bar{x}_{T_{n}}^{*}$ is calculated as the solution of PDE (2.6) with the survival probability of bank runs $\theta\left(T_{n+1}, x\right)$ at time $T_{n+1}$.

(ii) Compute the survival probability of bank runs as

$$
\theta\left(T_{n}, x\right)=\mathbf{1}_{\left\{x \geq \bar{x}_{T_{n}}^{I l l}\right\}}
$$

Algorithm 5.2 demonstrates the mechanism of our bank run model incorporating the feedback effect on the creditor's beliefs about the survival probability of bank runs. 


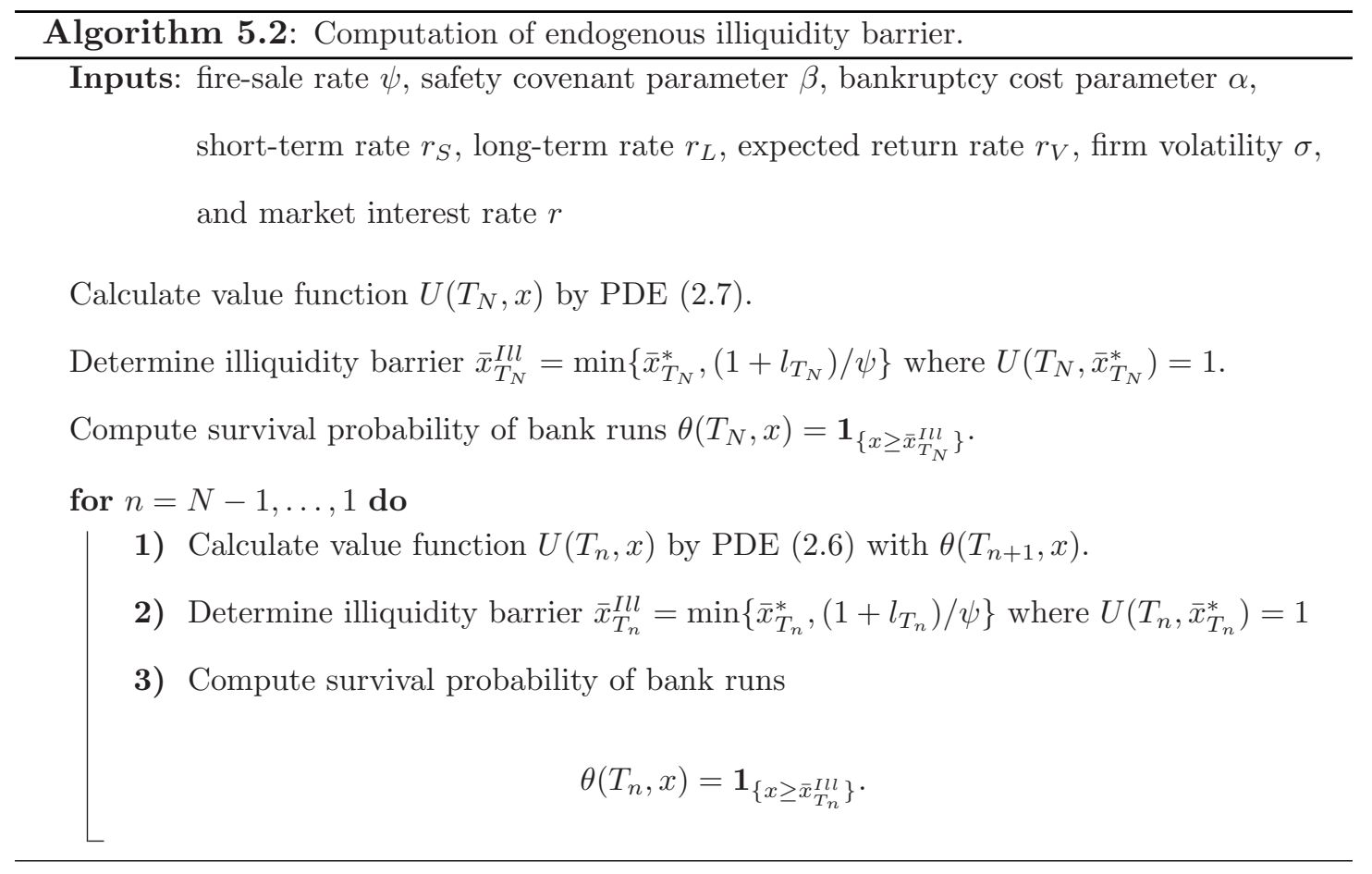

\section{Discussion and Conclusion}

In this paper, we provide a rigorous formulation for a class of structural credit risk models that take into account not only insolvency risk but also illiquidity risk due to possible bank runs. We show that there exists a unique threshold strategy, i.e., a bank run barrier for short-term creditors to decide when to withdraw their funding. This allows us to decompose the total credit risk into an illiquidity component based on the endogenous bank run barrier and an insolvency component based on the exogenous insolvency barrier. Our approach is based on the DDP and represents a dynamic bank run model that incorporates both discrete and staggered tenor structures for short-term debt. In this aspect, our paper is closely related to the existing dynamic bank run models of He and Xiong (2012b), Morris and Shin (2010), Liang et al. (2012), and Cheng and 
Milbradt (2012). The main characteristics of these models are summarized in Table 5 and are further discussed in the following.

Table 5: Comparisons of dynamic bank run models

\begin{tabular}{cccccc}
\hline & \multicolumn{2}{c}{ He\&Xiong } & Morris\&Shin Liang et al & Cheng\&Milbradt & Current paper \\
\hline \hline Time horizon & infinite & two periods & finite & infinite & finite \\
\hline Tenor structure & Poisson & No & discrete & Poisson & discrete/Cox \\
\hline Decision makers & creditors & creditors & creditors & creditors/equity holders & creditors/equity holders \\
\hline Dynamics & DPP & No & DPP & DPP & DPP/optimal stopping time \\
\hline Beliefs & endogenous & exogenous & exogenous & endogenous & exogenous/endogenous \\
\hline Equilibrium & general & partial & partial & general & partial/general \\
\hline
\end{tabular}

In dynamic bank run models, one crucial assumption is the maturity structure of short-term debt. Both He and Xiong (2012b) and Cheng and Milbradt (2012) utilize the Poisson random maturity assumption to capture the staggered tenor structure, whereas Liang et al. (2012) assume a sequence of deterministic rollover dates generalizing the two-period model of Morris and Shin (2010). In this paper, we consider both discrete and staggered tenor structures. Moreover, we show that the two tenor structures converge to each other when the rollover frequency goes to infinity.

The default mechanism in dynamic bank run models is mainly triggered by creditors' runs as shown in He and Xiong (2012b), Morris and Shin (2010), and Liang et al. (2012). This is different from traditional structural credit risk models where the default mechanism is usually triggered by equity holders as they either exogenously set a default barrier or endogenously determine an optimal default barrier ${ }^{5}$. Cheng and Milbradt (2012) consider decision problems of both creditors and equity holders in the dynamic

\footnotetext{
${ }^{5}$ Structural credit risk models with an exogenous default barrier are also dubbed first-passage-time
} 
bank run setting. In this paper, we consider that the equity holders exogenously set the insolvency barrier, while the creditors endogenously determine the bank run barrier and the illiquidity barrier. Most of dynamic bank run models are based on the DPP. In this paper, we further prove that the DPP is in fact derived from an optimal stopping time problem with control constraints.

Last but not least, the representative creditor's beliefs about other creditors' behavior also characterizes a dynamic bank run model. In Morris and Shin (2010) and Liang et al. (2012) such beliefs are modeled exogenously and therefore lead to a partial equilibrium. In this paper, besides such exogenous beliefs, we also consider the feedback effect of the bank run barrier on the representative creditor's beliefs about other creditors' behavior. In this aspect, our model represents a general equilibrium model in the same spirit as He and Xiong (2012b) and Cheng and Milbradt (2012).

\section{Appendix}

\section{A Proofs}

\section{A.1 Proof of Proposition 2.2}

The proof is essentially the same as Lemma 3.2 and 3.3 in Liang and Jiang (2012), so we only sketch it.

models as in Black and Cox (1976), Longstaff and Schwartz (1995), and Briys and de Varenne (1997), among others. If the default barrier is determined endogenously by equity holders, the models are also referred as optimal capital structure models, which were initiated by Leland $(1994,1998)$ and Leland and Toft (1996), followed by Hilberink and Rogers (2002) and Chen and Kou (2009), among others. 
First, note that under the new coordinate $\bar{x}=x /\left(\beta l_{t}\right)$, the PDEs (2.6) and (2.7) become $\mathcal{L}^{v} W_{n}=0$ on a regular domain $\left[T_{n}, T_{n+1}\right] \times[1, \infty)$. The Green's function $\mathbb{G}\left(t, \bar{x} ; T_{n+1}, \xi\right)$ for the operator $\mathcal{L}^{v}$ on $\left[T_{n}, T_{n+1}\right] \times[1, \infty)$ is the solution to the following PDE problem

$$
\left\{\begin{array}{l}
\mathcal{L}^{v} \mathbb{G}\left(t, \bar{x} ; T_{n+1}, \xi\right)=0 \\
\left.\mathbb{G}\right|_{\bar{x}=1}=0 \\
\left.\mathbb{G}\right|_{t=T_{n+1}}=\delta(\bar{x}-\xi) .
\end{array}\right.
$$

By making the transformation $y=\log (\bar{x} / \xi), \tau=T_{n+1}-t$, and

$$
\begin{aligned}
& \mathbb{G}\left(\tau, y ; T_{n+1}, \xi\right) \\
= & \exp \left\{\left[r_{S}-r-\frac{1}{2 \sigma^{2}}\left(r_{V}-r_{L}-\frac{\sigma^{2}}{2}\right)^{2}\right] \tau-\frac{1}{\sigma^{2}}\left(r_{V}-r_{L}-\frac{\sigma^{2}}{2}\right) y\right\} \mathbb{H}\left(\tau, y ; T_{n+1}, \xi\right),
\end{aligned}
$$

it is easy to verify that $\mathbb{H}\left(\tau, y ; T_{n+1}, \xi\right)$ satisfies a heat equation on the half plane. Its solution can be easily obtained by the standard image method.

Next, given the Green's function $\mathbb{G}\left(\tau, y ; T_{n+1}, \xi\right)$, we derive the solution to $\mathcal{L}^{v} W_{n}=0$ on the domain $\left[T_{n}, T_{n+1}\right] \times[1, \infty)$ with the boundary and terminal data $P_{n}$ and $Q_{n}$ by applying integration by parts. Consider the adjoint problem of $($ A.1 $)$ on $\left[t, T_{n+1}\right] \times[1, \infty)$

$$
\left\{\begin{array}{l}
\hat{\mathcal{L}}^{v} \hat{\mathbb{G}}(\eta, \xi ; t, \bar{x})=0 \\
\left.\hat{\mathbb{G}}\right|_{\xi=1}=0 \\
\left.\hat{\mathbb{G}}\right|_{\eta=t}=\delta(\xi-\bar{x}),
\end{array}\right.
$$

where $\hat{\mathcal{L}}^{v}$ is the adjoint operator of $\mathcal{L}^{v}$

$$
\hat{\mathcal{L}}^{v}=-\frac{\partial}{\partial \eta}+\frac{1}{2} \sigma^{2} \frac{\partial^{2}}{\partial \xi^{2}} \xi^{2}-\left(r_{V}-r_{L}\right) \frac{\partial}{\partial \xi} \xi+\left(r_{S}-r\right)
$$

Since $W_{n}(\eta, \xi)$ satisfies $\mathcal{L}^{v} W_{n}=0$ and $\hat{G}(\eta, \xi ; t, \bar{x})$ satisfies the adjoint equation $\hat{\mathcal{L}}^{v} \hat{G}(\eta, \xi ; t, \bar{x})=$ 0 , applying integration by parts to the integral

$$
\int_{1}^{\infty} d \xi \int_{t+\epsilon}^{T_{n+1}-\epsilon}\left[\hat{G}(\eta, \xi ; t, \bar{x}) \mathcal{L}^{v} W_{n}(\eta, \xi)-W_{n}(\eta, \xi) \hat{\mathcal{L}}^{v} \hat{G}(\eta, \xi ; t, \bar{x})\right] d \eta
$$


and using the boundary and terminal data $P_{n}$ and $Q_{n}$ for $W_{n}(\eta, \xi)$ will give us the Green's representation formula (2.8).

\section{A.2 Proof of Proposition 3.2}

We have the following properties for the first arrival time (i.e., the first short-term debt maturity) $T_{1}$ and the intensity $g\left(X_{t}\right)$, the proof of which can be found for example in Bielecki and Rutkowski (2002).

Lemma A.1 The process $\Gamma$ defined by $\Gamma_{t}=\int_{0}^{t} g\left(X_{s}\right)$ ds for $t \geq 0$ is an $\mathcal{F}_{t}$-hazard process associated with $T_{1}$, that is,

$$
\Gamma_{t}=-\log \mathbf{Q}\left(T_{1}>t \mid \mathcal{F}_{t}\right)=-\log \mathbf{Q}\left(T_{1}>t \mid \mathcal{F}_{\infty}\right)
$$

Moreover, for any $\mathcal{F}_{t}$-adapted process $Y_{t}$ and $\mathcal{F}_{t}$-stopping time $\tau$, on the event $\left\{T_{1}>t\right\}$,

$$
\begin{gathered}
\mathbf{E}\left[\mathbf{1}_{\left\{T_{1} \geq \tau\right\}} Y_{\tau} \mid \mathcal{F}_{t}\right]=\mathbf{E}\left[Y_{\tau} e^{-\int_{t}^{\tau} g\left(X_{s}\right) d s} \mid \mathcal{F}_{t}\right] . \\
\mathbf{E}\left[\mathbf{1}_{\left\{t<T_{1}<\tau\right\}} Y_{T_{1}} \mid \mathcal{F}_{t}\right]=\mathbf{E}\left[\int_{t}^{\tau} Y_{s} e^{-\int_{t}^{s} g\left(X_{u}\right) d u} g\left(X_{s}\right) d s \mid \mathcal{F}_{t}\right] .
\end{gathered}
$$

In the following, we employ the distribution of $T_{1}$ given by Lemma A.1 to calculate (3.2). For the first and the third terms, by using (A.4), we obtain

$$
\begin{aligned}
& \mathbf{E}_{t}^{x}\left\{\mathbf{1}_{\left\{t<T_{1}<\tau^{I n s} \wedge T\right\}} e^{\left(r_{S}-r\right)\left(T_{1}-t\right)}\left[\theta\left(X_{T_{1}}\right) \max \left\{1, U\left(T_{1}, X_{T_{1}}\right)\right\}+\left(1-\theta\left(X_{T_{1}}\right)\right) R_{T_{1}}\right]\right\} \\
= & \mathbf{E}_{t}^{x}\left\{\int_{t}^{\tau^{I n s} \wedge T} e^{\int_{t}^{s}\left(r_{S}-r-g\left(X_{u}\right)\right) d u} g\left(X_{s}\right)\right. \\
& \left.\times\left[\theta\left(X_{s}\right) \max \left\{1, U\left(s, X_{s}\right)\right\}+\left(1-\theta\left(X_{s}\right)\right) \alpha X_{s} /\left(1+l_{s}\right)\right] d s\right\} .
\end{aligned}
$$

For the second term, based on (A.3), we obtain

$$
\begin{aligned}
& \mathbf{E}_{t}^{x}\left\{\mathbf{1}_{\left\{T_{1} \geq \tau^{I n s}, t \leq \tau^{I n s}<T\right\}} e^{\left(r_{S}-r\right)\left(\tau^{I n s}-t\right)} R_{\tau^{I n s}}\right\} \\
= & \mathbf{E}_{t}^{x}\left\{\mathbf{1}_{\left\{t \leq \tau^{I n s}<T\right\}} e^{\int_{t}^{\tau^{I n s}}\left(r_{S}-r-g\left(X_{u}\right)\right) d u} \alpha \beta l_{\tau^{I n s}} /\left(1+l_{\tau^{I n s}}\right)\right\} .
\end{aligned}
$$


For the last term, by employing (A.3) again, we obtain

$$
\begin{aligned}
& \mathbf{E}_{t}^{x}\left\{\mathbf{1}_{\left\{T_{1} \geq T, \tau^{I n s} \geq T\right\}} e^{\left(r_{S}-r\right)(T-t)} \min \left\{1, X_{T} /\left(1+l_{T}\right)\right\}\right\} \\
= & \mathbf{E}_{t}^{x}\left\{\mathbf{1}_{\left\{\tau^{I n s} \geq T\right\}} e^{\int_{t}^{T}\left(r_{S}-r-g\left(X_{u}\right)\right) d u} \min \left\{1, X_{T} /\left(1+l_{T}\right)\right\}\right\} .
\end{aligned}
$$

By combing the above three equalities, we finally derive

$$
\begin{aligned}
U(t, x)= & \mathbf{E}_{t}^{x}\left\{\int_{t}^{\tau^{I n s} \wedge T} e^{\int_{t}^{s}\left(r_{S}-r-g\left(X_{u}\right)\right) d u} g\left(X_{s}\right)\right. \\
& \times\left[\theta\left(X_{s}\right) \max \left\{1, U\left(s, X_{s}\right)\right\}+\left(1-\theta\left(X_{s}\right)\right) \alpha X_{s} /\left(1+l_{s}\right)\right] d s . \\
& +\mathbf{1}_{\left\{t \leq \tau^{I n s}<T\right\}} e^{\int_{t}^{\tau^{I n s}}\left(r_{S}-r-g\left(X_{u}\right)\right) d u} \alpha \beta l_{\tau^{I n s}} /\left(1+l_{\tau^{I n s}}\right) \\
& \left.+\mathbf{1}_{\left\{\tau^{I n s} \geq T\right\}} e^{\int_{t}^{T}\left(r_{S}-r-g\left(X_{u}\right)\right) d u} \min \left\{1, X_{T} /\left(1+l_{T}\right)\right\}\right\} .
\end{aligned}
$$

Then similar to Proposition 2.1, the Feynman-Kac formula gives us the PDE representation for the value function $U(t, x)$ under the Cox maturity structure as provided in Proposition 3.2.

\section{A.3 Proof of Theorem 4.1}

For $n=0,1, \ldots, N$, we consider a sequence of optimal stopping time problems

$$
V\left(T_{n}, x\right)=\sup _{\tau \in\left\{T_{n+1}, T_{n+2}, \ldots\right\} \backslash T_{*}} \mathbf{E}_{T_{n}}^{x}\left\{\mathbf{1}_{\left\{T_{n} \leq \tau^{I n s}<T\right\}} \cdot \mathcal{A}_{T_{n}, \tau^{I n s}}+\mathbf{1}_{\left\{\tau^{I n s} \geq T\right\}} \cdot \mathcal{A}_{T_{n}, T}\right\},
$$

where $\tau$ is an $\mathcal{F}_{t}$-stopping time taking value in $\left\{T_{n+1}, T_{n+2}, \ldots\right\} \backslash T_{*}$. Then the value of the optimal stopping time problem $(4.1)$ is given by $V(0, x)$, and we want to show that $V(0, x)=U(0, x)$

Obviously we have $V\left(T_{N}, x\right)=U\left(T_{N}, x\right)$, since there is no optimization problem involved in $V\left(T_{N}, x\right)$ which is

$$
V\left(T_{N}, x\right)=\mathbf{E}_{T_{N}}^{x}\left\{\mathbf{1}_{\left\{T_{N} \leq \tau^{I n s}<T\right\}} e^{\left(r_{S}-r\right)\left(\tau^{I n s}-T_{N}\right)} R_{\tau^{I n s}}+\mathbf{1}_{\left\{\tau^{I n s} \geq T\right\}} e^{\left(r_{S}-r\right)\left(T-T_{N}\right)} R_{T}\right\}
$$


The idea is to introduce a sequence of auxiliary optimal stopping time problems whose optimal stopping times are also permitted to stop at the initial time $T_{n}$.

$$
\hat{V}\left(T_{n}, x\right)=\sup _{\tau \in\left\{T_{n}, T_{n+1}, \ldots\right\} \backslash T_{*}} \mathbf{E}_{T_{n}}^{x}\left\{\mathbf{1}_{\left\{T_{n} \leq \tau^{I n s}<T\right\}} \cdot \mathcal{A}_{T_{n}, \tau^{I n s}}+\mathbf{1}_{\left\{\tau^{I n s} \geq T\right\}} \cdot \mathcal{A}_{T_{n}, T}\right\} .
$$

We have the following relationship between $\hat{V}$ and $V$

$$
\hat{V}\left(T_{n}, x\right)=\theta(x) \max \left\{1, V\left(T_{n}, x\right)\right\}+(1-\theta(x)) R_{T_{n}}, \quad \text { for } n=0,1, \ldots, N
$$

For $n=0,1, \ldots, N-1$, by taking conditional expectation on $\mathcal{F}_{T_{n+1}}$ in $V\left(T_{n}, x\right)$, we obtain

$$
\begin{aligned}
& V\left(T_{n}, x\right)=\sup _{\tau} \mathbf{E}_{T_{n}}^{x}\left\{\mathbf{E}[\underbrace{\mathbf{1}_{\left\{T_{n} \leq \tau^{I n s}<T\right\}} \cdot \mathcal{A}_{T_{n}, \tau^{I n s}}+\mathbf{1}_{\left\{\tau^{I n s} \geq T\right\}} \cdot \mathcal{A}_{T_{n}, T}}_{\equiv I} \mid \mathcal{F}_{T_{n+1}}]\right\} \\
& =\sup _{\tau} \mathbf{E}_{T_{n}}^{x}\left\{\mathbf{E}\left[\left(\mathbf{1}_{\left\{T_{n} \leq \tau^{\text {Ins }}<T_{n+1}\right\}}+\mathbf{1}_{\left\{\tau^{\text {Ins }} \geq T_{n+1}\right\}}\right) \times I \mid \mathcal{F}_{T_{n+1}}\right]\right\} \\
& =\sup _{\tau} \mathbf{E}_{T_{n}}^{x}\left\{\mathbf{1}_{\left\{T_{n} \leq \tau^{I n s}<T_{n+1}\right\}} e^{\left(r_{S}-r\right)\left(\tau^{I n s}-T_{n}\right)} R_{\tau^{I n s}}\right. \\
& \left.+\mathbf{1}_{\left\{\tau^{I n s} \geq T_{n+1}\right\}} e^{\left(r_{S}-r\right)\left(T_{n+1}-T_{n}\right)} \mathbf{E}\left[\mathbf{1}_{\left\{T_{n+1} \leq \tau^{I n s}<T\right\}} \cdot \mathcal{A}_{T_{n+1}, \tau^{I n s}}+\mathbf{1}_{\left\{\tau^{I n s} \geq T\right\}} \cdot \mathcal{A}_{T_{n+1}, T} \mid \mathcal{F}_{T_{n+1}}\right]\right\} \\
& =\sup _{\tau} \mathbf{E}_{T_{n}}^{x}\left\{\mathbf{1}_{\left\{T_{n} \leq \tau^{I n s}<T_{n+1}\right\}} e^{\left(r_{S}-r\right)\left(\tau^{I n s}-T_{n}\right)} R_{\tau^{I n s}}\right. \\
& \left.+\mathbf{1}_{\left\{\tau^{I n s} \geq T_{n+1}\right\}} e^{\left(r_{S}-r\right)\left(T_{n+1}-T_{n}\right)} \mathbf{E}_{T_{n+1}}^{X_{T_{n+1}}}\left[\mathbf{1}_{\left\{T_{n+1} \leq \tau^{I n s}<T\right\}} \cdot \mathcal{A}_{T_{n+1}, \tau^{I n s}}+\mathbf{1}_{\left\{\tau^{I n s} \geq T\right\}} \cdot \mathcal{A}_{T_{n+1}, T}\right]\right\},
\end{aligned}
$$

where we used the Markovian property for $X$ in the last equality. Note that the first term in the bracket does not involve the stopping time $\tau$, so the supremum over $\tau$ only takes action on the second term and $V\left(T_{n}, x\right)$ is equal to

$$
\begin{aligned}
\mathbf{E}_{T_{n}}^{x} & \left\{\mathbf{1}_{\left\{T_{n} \leq \tau^{I n s}<T_{n+1}\right\}} e^{\left(r_{S}-r\right)\left(\tau^{I n s}-T_{n}\right)} R_{\tau^{I n s}}+\mathbf{1}_{\left\{\tau^{I n s} \geq T_{n+1}\right\}} e^{\left(r_{S}-r\right)\left(T_{n+1}-T_{n}\right)}\right. \\
& \left.\times \sup _{\tau \in\left\{T_{n+1}, T_{n+2}, \ldots\right\} \backslash T_{*}} \mathbf{E}_{T_{n+1}}^{X_{T_{n+1}}}\left[\mathbf{1}_{\left\{T_{n+1} \leq \tau^{I n s}<T\right\}} \cdot \mathcal{A}_{T_{n+1}, \tau^{I n s}}+\mathbf{1}_{\left\{\tau^{I n s} \geq T\right\}} \cdot \mathcal{A}_{T_{n+1}, T}\right]\right\},
\end{aligned}
$$

which, according to the definition of $\hat{V}$, is

$\mathbf{E}_{T_{n}}^{x}\left\{\mathbf{1}_{\left\{T_{n} \leq \tau^{I n s}<T_{n+1}\right\}} e^{\left(r_{S}-r\right)\left(\tau^{I n s}-T_{n}\right)} R_{\tau^{I n s}}+\mathbf{1}_{\left\{\tau^{I n s} \geq T_{n+1}\right\}} e^{\left(r_{S}-r\right)\left(T_{n+1}-T_{n}\right)} \hat{V}\left(T_{n+1}, X_{T_{n+1}}\right)\right\}$. 
By the relationship (A.5), we obtain the recursive formulation for $V\left(T_{n}, x\right)$ :

$$
\begin{aligned}
V\left(T_{n}, x\right)= & \mathbf{E}_{T_{n}}^{x}\left\{\mathbf{1}_{\left\{T_{n} \leq \tau^{I n s}<T_{n+1}\right\}} e^{\left(r_{S}-r\right)\left(\tau^{I n s}-T_{n}\right)} R_{\tau^{I n s}}\right. \\
& \left.+\mathbf{1}_{\left\{\tau^{I n s} \geq T_{n+1}\right\}} e^{\left(r_{S}-r\right)\left(T_{n+1}-t\right)}\left[\theta\left(X_{T_{n+1}}\right) \max \left\{1, V\left(T_{n+1}, X_{T_{n+1}}\right)\right\}+\left(1-\theta\left(X_{T_{n+1}}\right)\right) R_{T_{n+1}}\right]\right\} .
\end{aligned}
$$

This is just the dynamic programming equation for $U(t, x)$ in $(2.5)$. Since we already proved $V\left(T_{N}, x\right)=U\left(T_{N}, x\right)$, by proceeding backwards we obtain $V(0, x)=U(0, x)$.

\section{A.4 Proof of Theorem 4.2}

The proof is essentially the same as the proof for Theorem 4.1. For any $t \geq 0$, by letting $X$ start from $X_{t}=x$ and $\left\{T_{n}\right\}_{n \geq 0}$ start from $T_{0}=t$, we consider a family of optimal stopping problems

$$
V(t, x)=\sup _{\tau \in\left\{T_{n}\right\}_{n \geq 1} \backslash T_{*}} \mathbf{E}_{t}^{x}\left\{\mathbf{1}_{\left\{t \leq \tau^{I n s}<T\right\}} \cdot \mathcal{A}_{t, \tau^{I n s}}+\mathbf{1}_{\left\{\tau^{I n s} \geq T\right\}} \cdot \mathcal{A}_{t, T}\right\}
$$

where $\tau$ is a $\mathcal{G}_{t^{-}}$-stopping time taking value in $\left\{T_{n}\right\}_{n \geq 1} \backslash T_{*}$. Therefore, $\tau$ is not allowed to stop at the starting time $t$. The value of the optimal stopping time problem (4.2) is given by $V(0, x)$, and we want to prove that $V(0, x)=U(0, x)$.

Similarly to the case of the discrete tenor structure, we introduce a family of auxiliary optimal stopping time problems where the optimal stopping times are also allowed to stop at the starting time $t$

$$
\hat{V}(t, x)=\sup _{\tau \in\left\{T_{n}\right\}_{n \geq 0} \backslash T_{*}} \mathbf{E}_{t}^{x}\left\{\mathbf{1}_{\left\{t \leq \tau^{I n s}<T\right\}} \cdot \mathcal{A}_{t, \tau^{I n s}}+\mathbf{1}_{\left\{\tau^{I n s} \geq T\right\}} \cdot \mathcal{A}_{t, T}\right\}
$$

We have the following relationship between $\hat{V}$ and $V$

$$
\hat{V}(t, x)=\theta(x) \max \{1, V(t, x)\}+(1-\theta(x)) R_{t} \quad \text { for } t \in[0, T) .
$$


Taking expectations conditional on $\mathcal{F}_{T_{1}}$ in $V(t, x)$ and using the strong Markov property for $X$, we obtain for any $t \in[0, T)$

$$
\begin{aligned}
V(t, x)= & \sup _{\tau} \mathbf{E}_{t}^{x}\left\{\mathbf { E } \left[\left(\mathbf{1}_{\left\{t<T_{1}<\tau^{I n s}\right\}}+\mathbf{1}_{\left\{T_{1} \geq \tau^{I n s}\right\}}\right) \mathbf{1}_{\left\{t \leq \tau^{I n s}<T\right\}} \cdot \mathcal{A}_{t, \tau^{I n s}}\right.\right. \\
& \left.\left.+\left(\mathbf{1}_{\left\{t<T_{1}<T\right\}}+\mathbf{1}_{\left\{T_{1} \geq T\right\}}\right) \mathbf{1}_{\left\{\tau^{I n s} \geq T\right\}} \cdot \mathcal{A}_{t, T} \mid \mathcal{F}_{T_{1}}\right]\right\} \\
= & \sup _{\tau} \mathbf{E}_{t}^{x}\left\{\mathbf{E}\left[\mathbf{1}_{\left\{t \leq \tau^{I n s}<T\right\}} \mathbf{1}_{\left\{t<T_{1}<\tau^{I n s}\right\}} \mathcal{A}_{t, \tau^{I n s}} \mid \mathcal{F}_{T_{1}}\right]+\mathbf{1}_{\left\{t \leq \tau^{I n s}<T\right\}} \mathbf{1}_{\left\{T_{1} \geq \tau^{I n s}\right\}} e^{\left(r_{S}-r\right)\left(\tau^{I n s}-t\right)} R_{\tau^{I n s}}\right. \\
& \left.+\mathbf{E}\left[\mathbf{1}_{\left\{\tau^{I n s} \geq T\right\}} \mathbf{1}_{\left\{t<T_{1}<T\right\}} \mathcal{A}_{t, T} \mid \mathcal{F}_{T_{1}}\right]+\mathbf{1}_{\left\{\tau^{I n s} \geq T\right\}} \mathbf{1}_{\left\{T_{1} \geq T\right\}} e^{\left(r_{S}-r\right)(T-t)} R_{T}\right\} \\
= & \sup _{\tau} \mathbf{E}_{t}^{x}\left\{\mathbf{1}_{\left\{t<T_{1}<\tau^{I n s} \wedge T\right\}} e^{\left(r_{S}-r\right)\left(T_{1}-t\right)} \mathbf{E}\left[\mathbf{1}_{\left\{T_{1} \leq \tau^{I n s}<T\right\}} \mathcal{A}_{T_{1}, \tau^{I n s}}+\mathbf{1}_{\left\{\tau^{I n s} \geq T\right\}} \mathcal{A}_{T_{1}, T} \mid \mathcal{F}_{T_{1}}\right]\right. \\
& \left.+\mathbf{1}_{\left\{t \leq \tau^{I n s}<T\right\}} \mathbf{1}_{\left\{T_{1} \geq \tau^{I n s}\right\}} e^{\left(r_{S}-r\right)\left(\tau^{I n s}-t\right)} R_{\tau^{I n s}}+\mathbf{1}_{\left\{\tau^{I n s} \geq T\right\}} \mathbf{1}_{\left\{T_{1} \geq T\right\}} e^{\left(r_{S}-r\right)(T-t)} R_{T}\right\} \\
= & \sup _{\tau} \mathbf{E}_{t}^{x}\left\{\mathbf{1}_{\left\{t<T_{1}<\tau^{I n s} \wedge T\right\}} e^{\left(r_{S}-r\right)\left(T_{1}-t\right)} \mathbf{E}_{T_{1}}^{X_{T_{1}}}\left[\mathbf{1}_{\left\{T_{1} \leq \tau^{I n s}<T\right\}} \mathcal{A}_{T_{1}, \tau^{I n s}}+\mathbf{1}_{\left\{\tau^{I n s} \geq T\right\}} \mathcal{A}_{T_{1}, T}\right]\right. \\
& \left.+\mathbf{1}_{\left\{t \leq \tau^{I n s}<T\right\}} \mathbf{1}_{\left\{T_{1} \geq \tau^{I n s}\right\}} e^{\left(r_{S}-r\right)\left(\tau^{I n s}-t\right)} R_{\tau^{I n s}}+\mathbf{1}_{\left\{\tau^{I n s} \geq T\right\}} \mathbf{1}_{\left\{T_{1} \geq T\right\}} e^{\left(r_{S}-r\right)(T-t)} R_{T}\right\},
\end{aligned}
$$

which by the definition of $\hat{V}$ is equal to

$$
\begin{aligned}
\mathbf{E}_{t}^{x} & \left\{\mathbf{1}_{\left\{t<T_{1}<\tau^{I n s} \wedge T\right\}} e^{\left(r_{S}-r\right)\left(T_{1}-t\right)} \hat{V}\left(T_{1}, X_{T_{1}}\right)\right. \\
& \left.+\mathbf{1}_{\left\{t \leq \tau^{I n s}<T\right\}} \mathbf{1}_{\left\{T_{1} \geq \tau^{I n s}\right\}} e^{\left(r_{S}-r\right)\left(\tau^{I n s}-t\right)} R_{\tau^{I n s}}+\mathbf{1}_{\left\{\tau^{I n s} \geq T\right\}} \mathbf{1}_{\left\{T_{1} \geq T\right\}} e^{\left(r_{S}-r\right)(T-t)} R_{T}\right\} .
\end{aligned}
$$

The result then follows from the relationship (A.6).

\section{B Numerical approximation of the solution to PDE (3.3)}

We first transform $\operatorname{PDE}(3.3)$ by defining $y=\log \left(x / \beta l_{t}\right), \tau=T-t$ and $u(\tau, y)=$ $U(t, x)$. Then PDE (3.3) reduces to

$$
\frac{\partial u}{\partial \tau}=\frac{1}{2} \sigma^{2} \frac{\partial^{2} u}{\partial y^{2}}+\left(r_{V}-r_{L}-\frac{1}{2} \sigma^{2}\right) \frac{\partial u}{\partial y}+\left(r_{s}-r-\zeta\right) u+\eta \max \{1, u\}+\kappa,
$$


where

$$
\zeta(\tau, y)=g(x) ; \eta(\tau, y)=g(x) \theta(x) ; \kappa(\tau, y)=g(x)(1-\theta(x)) \alpha x /\left(1+l_{t}\right)
$$

with boundary and initial conditions

$$
\begin{aligned}
& u(\tau, 0)=\alpha \beta l_{T-\tau} /\left(1+l_{T-\tau}\right)=P(\tau)=P \\
& u(0, y)=\min \left\{1, e^{y} \beta l_{T} /\left(1+l_{T}\right)\right\}=\Phi(y)=\Phi .
\end{aligned}
$$

In the following, we derive the implicit finite difference equation for PDE (B.1). Let $\Delta \tau$ denote the step size between two updates of the value function $u$ in the time dimension. Similarly, $\Delta y$ denotes the step size between grid points in the space dimension of the value function $u$. The relevant range of two variables is taken to be

$$
(\tau, y) \in[0, T] \times[0, \bar{y}]
$$

where $\bar{y}$ is a large constant such that realization of $y$ outside the region $[0, \bar{y}]$ occurs with negligible probability. At each grid point, we define

$$
u_{j}^{n}=u(n \Delta \tau, j \Delta y)
$$

and the implicit finite difference equation for $(B .1)$ is

$$
\begin{aligned}
\frac{u_{j}^{n+1}-u_{j}^{n}}{\Delta \tau}= & \frac{1}{2} \sigma^{2} \frac{u_{j+1}^{n+1}-2 u_{j}^{n+1}+u_{j-1}^{n+1}}{\Delta y^{2}}+\left(r_{V}-r_{L}-\frac{1}{2} \sigma^{2}\right) \frac{u_{j+1}^{n+1}-u_{j-1}^{n+1}}{2 \Delta y} \\
& +\left(r_{s}-r-\zeta_{j}^{n+1}\right) u_{j}^{n+1}+\eta_{j}^{n+1} \max \left\{1, u_{j}^{n+1}\right\}+\kappa_{j}^{n+1},
\end{aligned}
$$

where

$$
\zeta_{j}^{n}=G(n \Delta \tau, j \Delta y) ; \eta_{j}^{n}=\eta(n \Delta \tau, j \Delta y) ; \kappa_{j}^{n}=F(n \Delta \tau, j \Delta y)
$$

for $0 \leq n \leq T / \Delta \tau$ and $0 \leq j \leq \bar{y} / \Delta y$. The corresponding boundary and initial conditions are

$$
u_{0}=P ; u_{\bar{y} / \Delta y}=0 ; u^{0}=\Phi,
$$


where $P$ and $\Phi$, with abuse of notation, denote the vectors containing the discrete values of the boundary and initial conditions, respectively.

The implicit finite difference equation (B.2) can be rewritten as the following nonlinear algebraic equation:

$$
A u^{n+1}-\left(\eta^{n+1}, \max \left\{1, u^{n+1}\right\}\right)=\bar{\kappa}^{n}
$$

where $\bar{\kappa}^{n}=\frac{1}{\Delta \tau} u^{n}+\kappa^{n}-[c P, 0,0, \ldots, 0]^{*}$, and $A$ is a tridiagonal matrix:

$$
A=\left(\begin{array}{ccccc}
a_{1} & b & 0 & \ldots & 0 \\
c & a_{2} & b & \ddots & 0 \\
0 & c & a_{3} & b & \ldots \\
0 & 0 & \ddots & \ddots & 0 \\
0 & 0 & \ldots & c & a_{\bar{y} / \Delta y-1}
\end{array}\right)
$$

with

$$
\begin{aligned}
a_{j} & =\frac{1}{\Delta \tau}+\frac{\sigma^{2}}{\Delta y^{2}}-\left(r_{S}-r-\zeta_{j}^{n+1}\right) ; \\
b & =-\frac{1}{2} \frac{\sigma^{2}}{\Delta y^{2}}-\frac{1}{2 \Delta y}\left(r_{V}-r_{L}-\frac{1}{2} \sigma^{2}\right), \\
c & =-\frac{1}{2} \frac{\sigma^{2}}{\Delta y^{2}}+\frac{1}{2 \Delta y}\left(r_{V}-r_{L}-\frac{1}{2} \sigma^{2}\right) .
\end{aligned}
$$

Finally, for $n=0,1, \ldots, T / \Delta \tau$, we use the standard Newton method to solve the nonlinear algebraic equation (B.3) as follows.

- Set $v^{1}=u^{n}$;

- For $m=1,2, \ldots$, solve $v^{m+1}$ recursively by the corresponding linear equation for

$$
A v^{m}-\left(\eta^{n+1}, \max \left\{1, v^{m}\right\}\right)-\bar{\kappa}^{n}+B_{m}^{n+1}\left(v^{m+1}-v^{m}\right)=0
$$


until sup $\left|v^{m+1}-v^{m}\right|<\epsilon$, where

$$
B_{m}^{n+1}=A-\eta^{n+1}\left(\begin{array}{ccccc}
\mathbf{1}_{\left\{v_{1}^{m}>1\right\}} & 0 & \ldots & 0 & 0 \\
0 & \mathbf{1}_{\left\{v_{2}^{m}>1\right\}} & 0 & \ldots & 0 \\
0 & 0 & \ddots & 0 & 0 \\
0 & 0 & 0 & 0 & \mathbf{1}_{\left\{v_{\bar{y} / \Delta y-1}^{m}>1\right\}}
\end{array}\right)
$$

- Suppose the above loop runs $M$ times. Then set $u^{n+1}=v^{M}$.

\section{References}

Adrian, T., and Shin, H.S. (2008): Liquidity and financial contagion. Financial Stability Review, Special issue on liquidity, No. 11, Banque de France.

Adrian, T., and Shin, H.S. (2010): Liquidity and leverage. Journal of Financial Intermediation 19(3), 418-437.

Bielecki, T.R., and Rutkowski, M. (2002): Credit risk: Modeling, valuation and hedging. Springer.

Black, F. and Cox, J. (1976): Some effects of bond indenture provisions. Journal of Finance 31, 351-367.

Briys, E. and de Varenne, F. (1997): Valuing risky fixed rate debt: An extension. Journal of Financial and Quantitative Analysis 32, 239-249.

Brunnermeier, M. (2009): Deciphering the liquidity and credit crunch 2007-08. Journal of Economic Perspectives 23, 77-100.

Chen, N. and Kou, S. (2009): Credit spread, implied volatility, and optimal capital 
structures with jump risk and endogenous defaults, Mathematical Finance, 19, 343378.

Cheng, I. H. and Milbradt, K. (2012): The hazards of debt: Rollover freezes, incentives, and bailouts. Review of Financial Studies 25(4), 1070-1110.

Diamond, D. and Dybvig, P. (1983): Bank runs, deposit insurance and liquidity. Journal of Political Economy 91, 401-419.

Ericsson, J. and Renault, O. (2006): Liquidity and credit risk. Journal of Finance 61, $2219-2250$.

Goldstein, I. and Pauzner, A. (2005): Demand-deposit contracts and the probability of bank runs. Journal of Finance 60, 1293-1327.

He, Z. and Xiong, W. (2012): Rollover risk and credit risk. Journal of Finance 67, $391-429$.

He, Z. and Xiong, W.(2012): Dynamic debt runs. Review of Financial Studies 25 (6), $1799-1843$

Hilberink, B. and Rogers, L.C.G. (2002): Optimal capital structure and endogenous default. Finance and Stochastics 6, 237-263.

Krylov, N.V. (2008): Controlled diffusion processes. Springer, 2nd printing edition.

Leland, H.E. (1994): Corporate debt value, bond covenants, and optimal capital structure. Journal of Finance 49, 1213-1252.

Leland, H.E. (1998): Agency costs, risk management, and capital structure. Journal of Finance 53, 1213-1243. 
Leland, H.E. and Toft, K. (1996): Optimal capital structure, endogenous bankruptcy, and the term structure of credit spreads. Journal of Finance 51, 987-1019.

Liang, G. and Jiang, L. (2012): A modified structural model for credit risk. IMA Journal of Management Mathematics 23, 147-170.

Liang, G., Lütkebohmert, E. and Xiao, Y. (Forthcoming): A multi-period bank run model for liquidirty risk. Review of Finance.

Longstaff, F. and Schwartz, E. (1995): A simple approach to valuing risky fixed and floating rate debt. Journal of Finance 50, 789-819.

Merton, R.C. (1974): On the pricing of corporate debt: The risk structure of interest rates. Journal of Finance 29, 449-470.

Morris, S. and Shin, H.S. (2010): Illiquidity component of credit risk. Working Paper. 


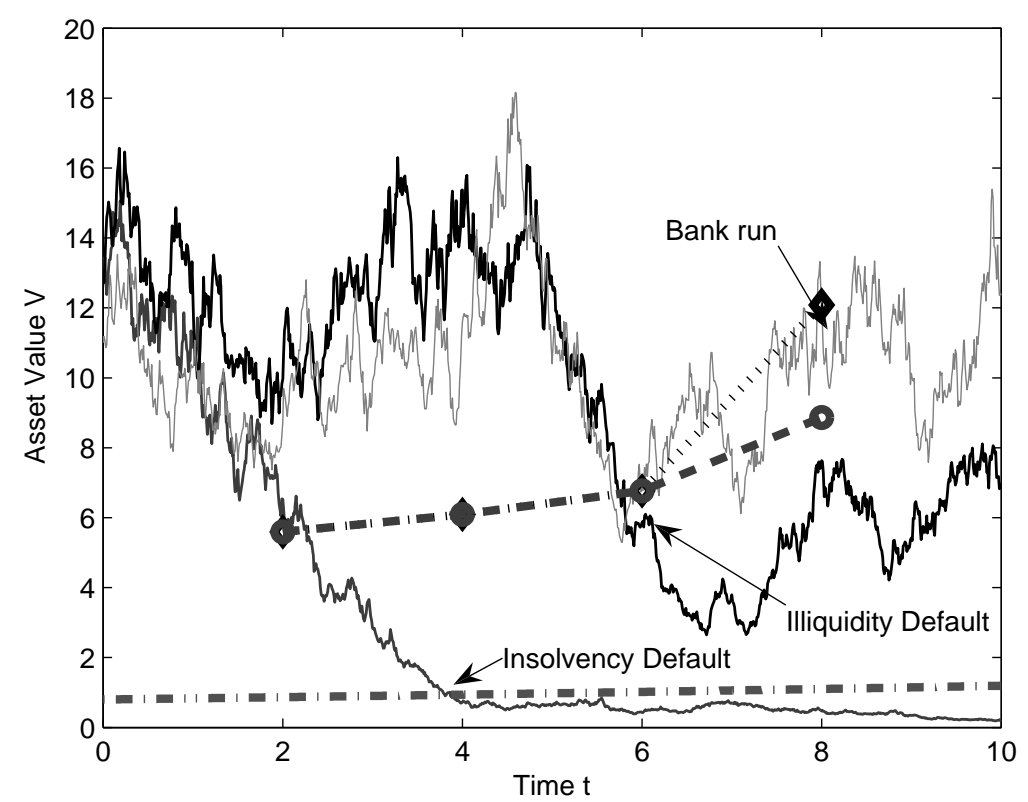

Figure 1: Scenario simulation with discrete tenor structure

The figure shows three simulated asset value paths in the model with a discrete tenor structure, where volatility $\sigma=0.4$, expected return rate $r_{V}=-0.02$, market interest rate $r=0.01$, shortterm rate $r_{S}=0.03$, and long-term rate $r_{L}=0.05$. The initial values of short- and long-term debt are set to $S_{0}=2$ and $L_{0}=2$, respectively. The safety covenant parameter $\beta=0.4$, the bankruptcy cost parameter $\alpha=0.6$, and the fire-sale rate is set to $\psi=0.6$. In this discrete tenor structure setting the number of rollover dates is set to $N=4$. The dotted line describes the bank run barrier, the dashed line the illiquidity barrier, and the dashed-dotted line the insolvency barrier. 


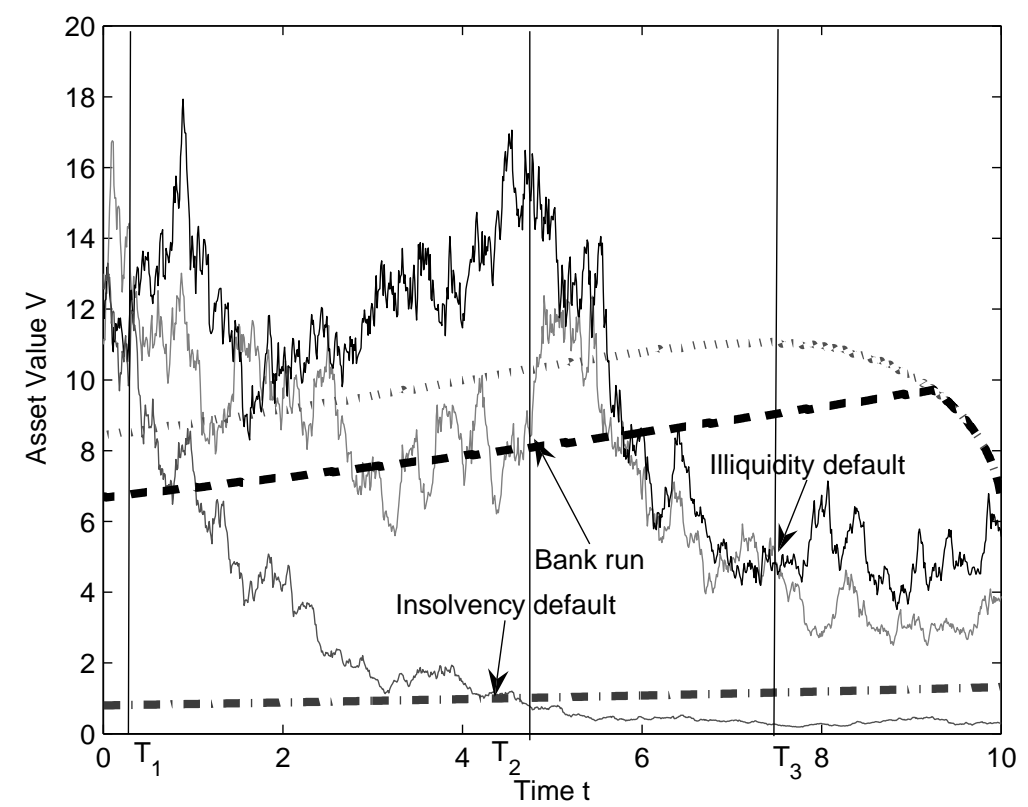

Figure 2: Scenario simulation with a staggered tenor structure

The figure shows three simulated asset value paths in the model with a staggered tenor structure where volatility $\sigma=0.4$, expected return rate $r_{V}=-0.02$, market interest rate $r=0.01$, shortterm rate $r_{S}=0.03$, and long-term rate $r_{L}=0.05$. The initial values of short- and long-term debt are set to $S_{0}=2$ and $L_{0}=2$, respectively. The safety covenant parameter $\beta=0.4$, the bankruptcy cost parameter $\alpha=0.6$, and the fire-sale rate is set to $\psi=0.6$. In this staggered tenor structure setting the intensity of the Cox process is chosen to be $g\left(X_{t}\right) \equiv 0.4$. The dotted line describes the bank run barrier, the dashed line the illiquidity barrier, and the dashed-dotted line the insolvency barrier. 


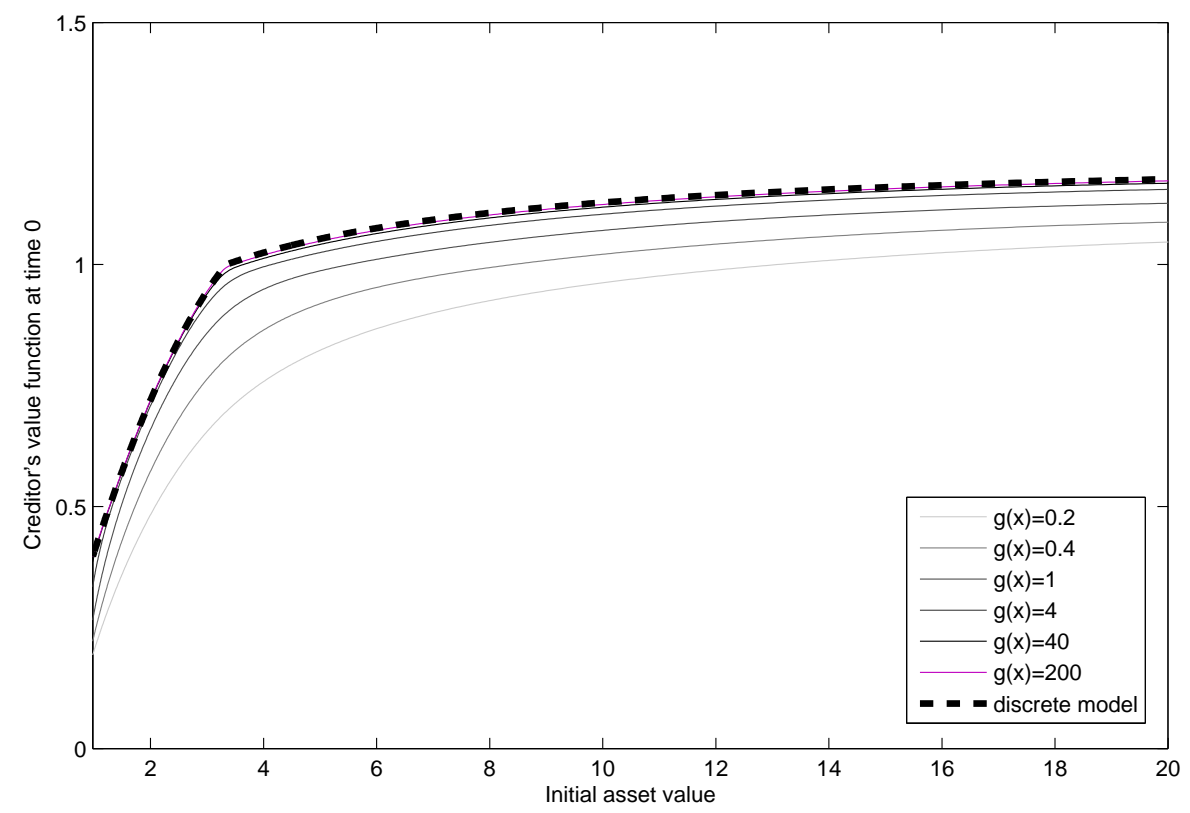

Figure 3: Influence of the intensity on creditor's value function

The figure shows the representative creditor's value function at time $t=0$ for increasing initial asset value $V_{0}$ in the discrete tenor structure model with $N=1000$ rollover dates and for the staggered tenor structure model for different intensities $g$ of the Cox process. Other parameters are the same as in Figure 2. 

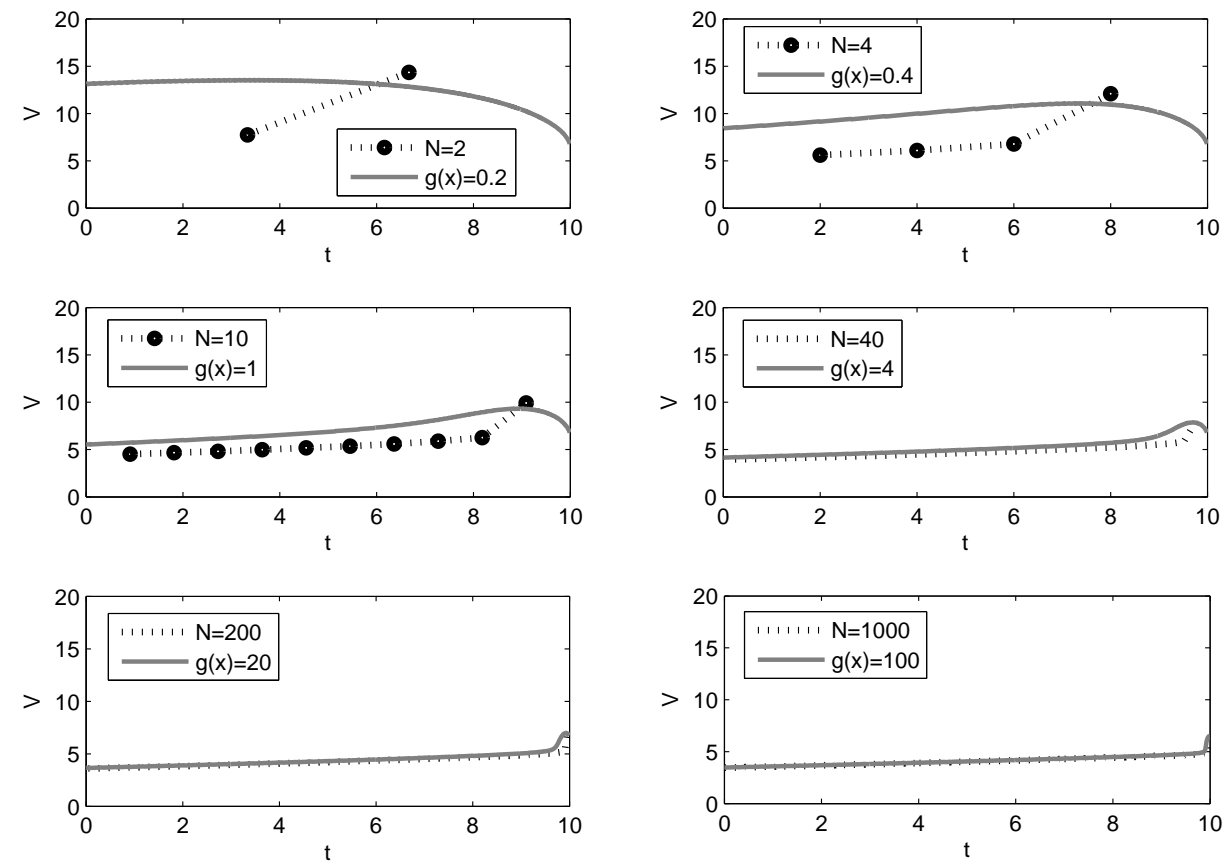

Figure 4: Comparison between the discrete and the staggered tenor structure models

The figure shows the bank run barrier depending on time $t$ for different rollover frequencies $N$ in the discrete tenor structure model and for different intensities $g(x)$ of the Cox process in the staggered tenor structure model. Other parameters are the same as in Figure 2. 